\title{
Méthodes de caractérisation des propriétés optiques non linéaires de conversion de fréquence des cristaux.
}

\author{
Benoît Boulanger et Jean-Philippe Fève \\ Laboratoire de Spectrométrie Physique (UMR CNRS 5588), \\ Université Joseph Fourier, \\ 140 avenue de la Physique, BP 87, \\ F-38402 SAINT MARTIN D'HËRES, FRANCE \\ benoit.boulanger@ujf-grenoble.fr \\ site web : http://www-lsp.ujf-grenoble.fr/
}

Résumé. Une partie importante des progrès réalisés au cours des dernières années sur les dispositifs de conversion de fréquence optique est due au développement de nouveaux cristaux à propriétés optiques non-linéaires et à la maîtrise croissante de leur qualité. La connaissance précise et fiable des propriétés d'un nouveau matériau à un stade précoce de son développement est primordiale pour choisir les cristaux dont la cristallogenèse mérite d'être poursuivie.

Le but de ce chapitre est de présenter les paramètres pertinents qui gouvernent les interactions optiques non linéaires de conversion de fréquence et les méthodes de caractérisation associées. Une comparaison des principaux cristaux convertisseurs de fréquence est donnée, ainsi que leurs domaines d'application.

\section{Mots-clés :}

42.65.Ky Harmonic generation, frequency conversion

42.70.Mp Nonlinear optical crystals

78.20.Ci Optical constants

42.65.An Optical susceptibility, hyperpolarizability

42.65.Yj Optical parametric oscillators and amplifiers 


\section{INTRODUCTION}

La conversion de fréquence optique est une propriété optique non linéaire qui est aujourd'hui à la base d'un grand nombre de dispositifs pour des applications très variées. Citons à titre d'exemple la spectroscopie, notamment pour la détection de gaz, mais également plusieurs techniques chirurgicales, ainsi que la télémétrie, la photo-lithographie ou la fusion thermonucléaire. Tous ces dispositifs paramétriques optiques associent une ou plusieurs sources lasers à un ou plusieurs cristaux à propriété optique non linéaire dans lesquels sont réalisées les mélanges de somme ou différence de fréquences

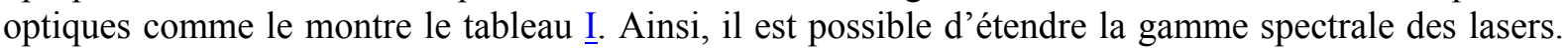
D'autre part, pour des longueurs d'onde choisies, ces interactions permettent de générer des faisceaux dont les caractéristiques seraient mieux adaptées que celles des sources lasers existantes vis-à-vis de l'application visée, en terme de durée d'impulsion, d'énergie, de fréquence de répétition, de qualités spatiales et spectrales. Parmi les dispositifs convertisseurs de fréquences, notons l'importance grandissante des oscillateurs paramétriques optiques (OPO) dont l'intérêt majeur est qu'ils permettent la génération d'un rayonnement laser accordable en longueur d'onde à partir d'un seul rayonnement laser monochromatique.

Il reste encore beaucoup à faire dans le domaine des dispositifs paramétriques optiques, notamment : l'élargissement de la gamme spectrale, vers l'ultraviolet et l'infrarouge moyen et lointain, l'extension au régime continu et aux très hautes cadences de répétition ou l'amélioration de la qualité des faisceaux émis par les OPO. La réalisation de ces objectifs nécessite d'abord une recherche sur de nouveaux cristaux à propriétés optiques non linéaires, d'où l'importance du travail sur les méthodes de caractérisation des propriétés optiques non linéaires de conversion de fréquence qui font l'objet de ce chapitre.

Il faut être capable de mesurer ces propriétés optiques avec précision sur des échantillons de petite taille, souvent de quelques millimètres, ces dimensions étant typiques des premiers stades de cristallogenèse d'un nouveau matériau jamais synthétisé jusqu'alors. Il est en effet important de pouvoir caractériser un nouveau cristal à ce stade car la mise au point d'un procédé permettant l'obtention de cristaux centimétriques de bonne qualité est souvent très difficile. Il faut donc savoir le plus rapidement possible si les recherches sur le cristal en question doivent être poursuivies ou abandonnées, d'où la nécessité de concevoir des méthodes de caractérisation adaptées.

L'objectif de ce chapitre est de faire le point sur les paramètres qu'il est important de mesurer ainsi que sur les méthodes de caractérisation correspondantes utilisées actuellement. Une comparaison des principaux cristaux convertisseurs de fréquence est donnée, ainsi que leurs domaines d'application.

\section{LES PRINCIPALES INTERACTIONS DE CONVERSION DE FRÉQUENCE OPTIQUE}

Les principaux couplages paramétriques optiques sont donnés dans la figure $\underline{1}$. Ce sont des interactions de somme ou différence de fréquences qui impliquent le plus souvent trois ondes, les couplages à quatre ondes étant beaucoup moins efficaces.

La génération de second harmonique est de loin l'interaction qui a conduit au plus grand nombre d'applications commercialisées. Les OPO constituent aujourd'hui un enjeu scientifique et technologique important, leur intérêt majeur étant qu'ils permettent la génération d'un rayonnement accordable en longueur d'onde à partir d'un laser de longueur d'onde fixe.

\section{QUELQUES NOTIONS SUR LA MODÉLISATION DES INTERACTIONS OPTIQUES NON LINÉAIRES}

Il n'est pas question ici de refaire un cours d'optique non linéaire. Le lecteur qui débute est invité à consulter un certain nombre d'ouvrages de base $[\underline{1}, \underline{2}, \underline{3}]$, de même que le chapitre d'optique non linéaire rédigé par J. Y. Courtois dans le volume 4 de cette même collection, dédié aux lasers et à leurs applications scientifiques et médicales [4]. Seul le cas des interactions à trois ondes est détaillé ici. 


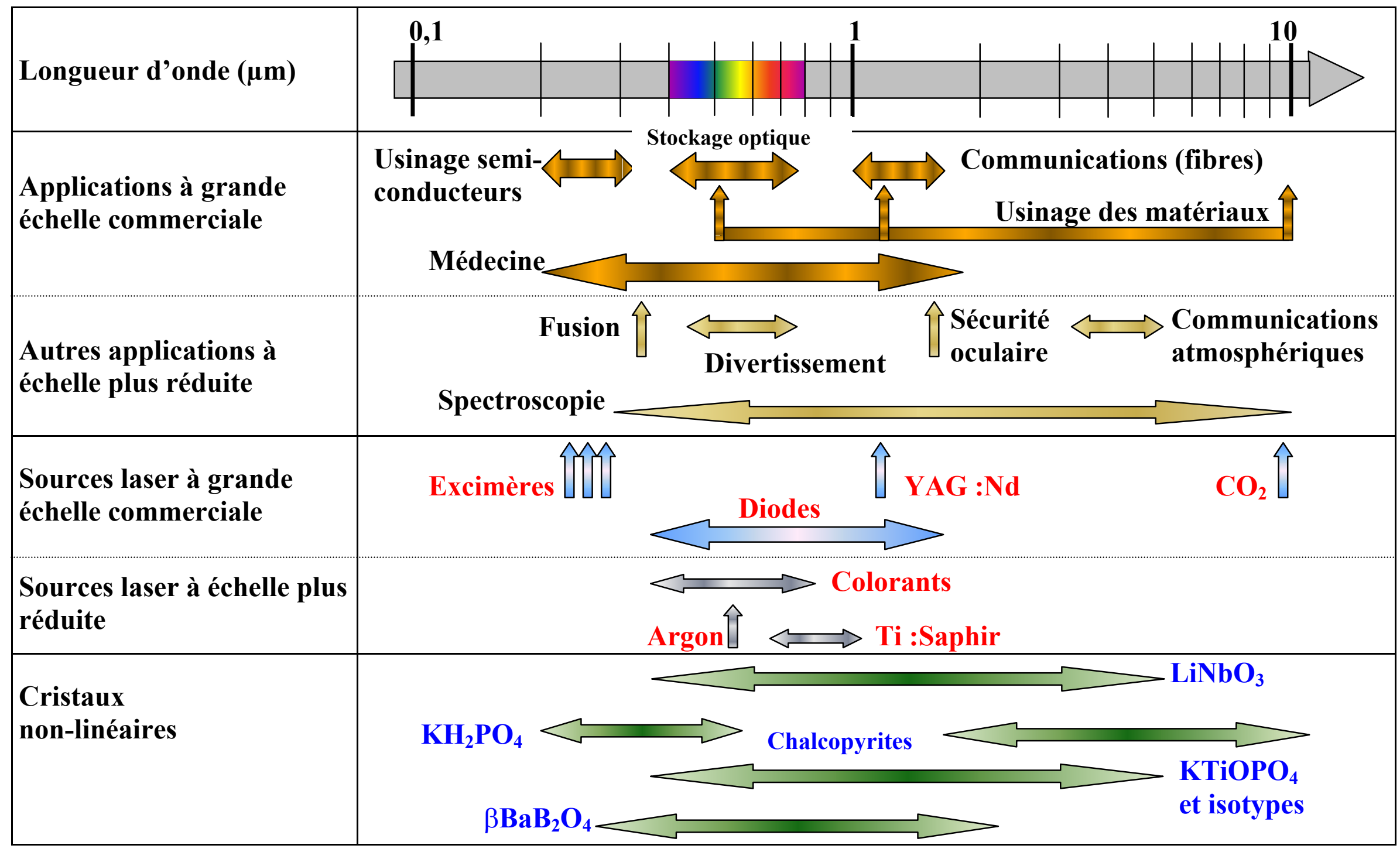

TAB. 1. Intérêt des cristaux convertisseurs de fréquence. 


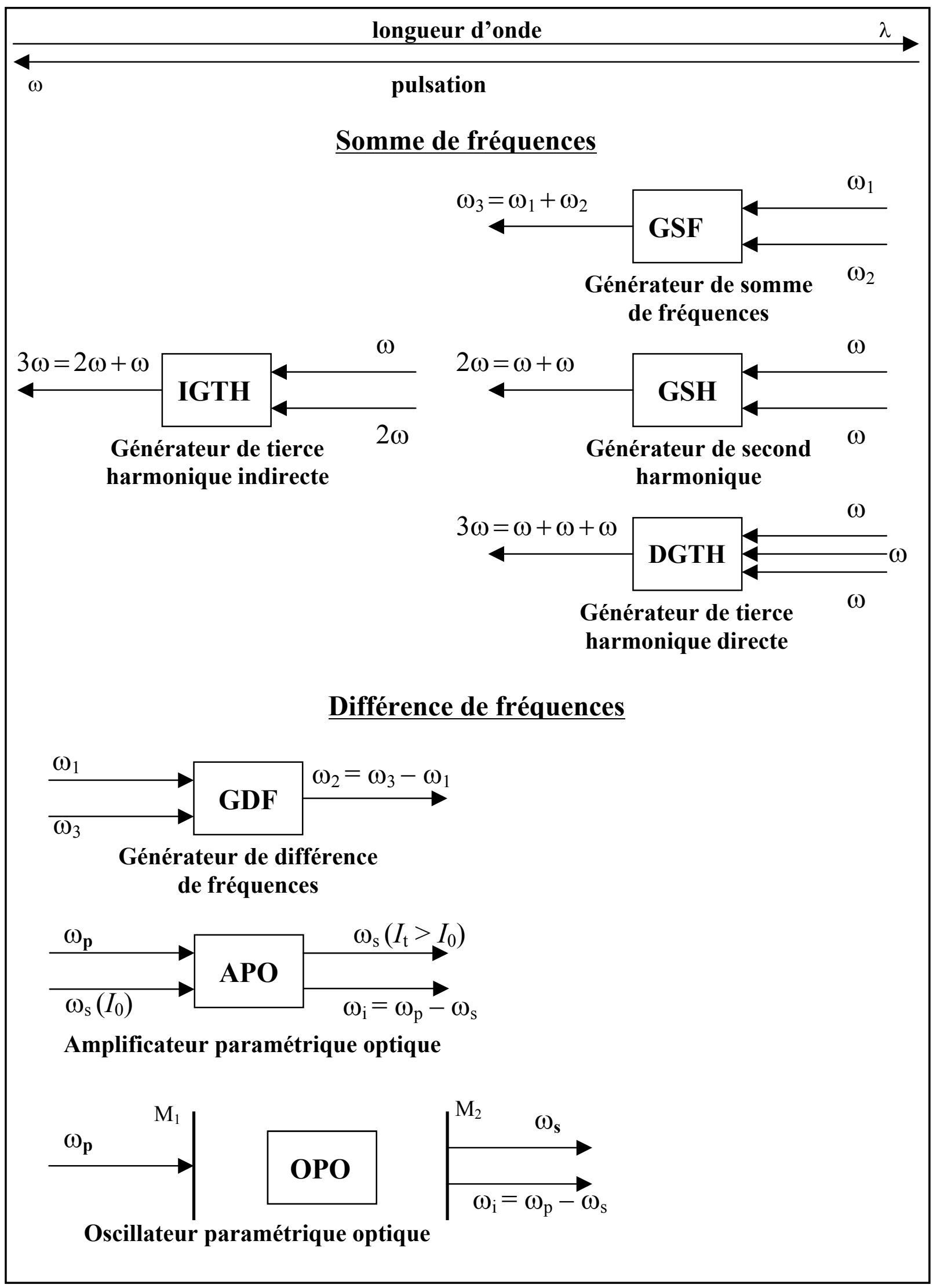

FIG. 1. Principales interactions de somme et différence de fréquences. $\omega, 2 \omega, 3 \omega, \omega_{1}, \omega_{2}, \omega_{3}, \omega_{\mathrm{p}}, \omega_{\mathrm{s}}$ et $\omega_{\mathrm{i}}$ sont les pulsations des ondes couplées. $I_{\mathrm{o}}$ et $I_{t}$ sont les intensités incidentes et transmises respectivement de l'onde de pulsation $\omega_{\mathrm{s}}$ qui est amplifiée dans l'amplificateur paramétrique optique (APO). Les miroirs $\mathrm{M}_{1}$ et $\mathrm{M}_{2}$ constituent la cavité de l'oscillateur paramétrique optique (OPO). 


\section{1. Équations régissant les interactions à trois ondes}

Nous considérons un cas simplifié, mais qui correspond néanmoins à un certain nombre de situations réelles et qui est suffisant pour définir les paramètres importants qui gouvernent tous les couplages paramétriques. Le champ d'approximation est le suivant: les trois ondes en interaction ont leurs vecteurs d'onde colinéaires, l'absorption est négligée, de même que la diffraction, la double réfraction et la dispersion des vitesses de groupe. Dans ce cadre, le système d'équations différentielles couplées régissant l'évolution de l'amplitude complexe des champs électriques des ondes en interaction s'écrit :

$$
\left\{\begin{array}{l}
\frac{\partial E_{1}}{\partial Z}=j \kappa_{1} E_{3} E_{2}{ }^{*} \exp (j \Delta k . Z) \\
\frac{\partial E_{2}}{\partial Z}=j \kappa_{2} E_{3} E_{1}{ }^{*} \exp (j \Delta k . Z) \\
\frac{\partial E_{3}}{\partial Z}=j \kappa_{3} E_{1} E_{2} \exp (-j \Delta k . Z)
\end{array}\right.
$$

- Les indices $i=1,2,3$ sont relatifs aux pulsations $\omega_{1}, \omega_{2}, \omega_{3}$ avec $\omega_{1}+\omega_{2}=\omega_{3}$; la pulsation est reliée à la longueur d'onde par $\omega_{i}=2 \pi c / \lambda_{i}$.

- $(X, Y, Z)$ est le repère du laboratoire où $Z$ est selon la direction de propagation des 3 ondes.

- $E_{i}=E_{i}(X, Y, Z)=A_{i}(X, Y, Z) \exp \left[j \Phi_{i}\right]$ est l'amplitude complexe du champ électrique de pulsation $\omega_{i}$.

- $\Delta k . Z$ est le déphasage entre la polarisation non linéaire et le champ rayonné, avec $\Delta k$ donné par : $\Delta k=k_{3}-\left(k_{1}+k_{2}\right)$ où $k_{\mathrm{i}}$ est le module du vecteur d'onde relatif à l'onde $i$ donné par $k_{i}=\left(\omega_{i} / c\right) n_{i}$, avec $n_{i}$ l'indice de réfraction à la pulsation $\omega_{i}$.

- $\kappa_{i}=\frac{\pi \chi_{\text {eff }}}{n_{i} \lambda_{i}}$ où $\lambda_{i}$ est la longueur d'onde et $\chi_{\text {eff }}$ le coefficient effectif. Dans le cas d'un milieu sans perte il est donné par :

$$
\chi_{e f f}=\mathbf{e}_{1} \cdot\left[\chi^{(2)}\left(\omega_{1}\right): \mathbf{e}_{3} \otimes \mathbf{e}_{2}^{*}\right]=\mathbf{e}_{2} \cdot\left[\chi^{(2)}\left(\omega_{2}\right): \mathbf{e}_{3} \otimes \mathbf{e}_{1}^{*}\right]=\mathbf{e}_{3} \cdot\left[\chi^{(2)}\left(\omega_{3}\right): \mathbf{e}_{1} \otimes \mathbf{e}_{2}\right]
$$

où $\mathbf{e}_{i}$ est le vecteur unitaire du champ électrique de l'onde $i$ et $\chi^{(2)}\left(\omega_{i}\right)$ est le tenseur de susceptibilité électrique de second ordre, qui est un tenseur polaire de rang 3 . Les symboles «®» et «:» correspondent respectivement au produit tensoriel et au produit contracté : compte tenu du rang des différents tenseurs polaires, les expressions entre crochets sont bien des tenseurs de rang 1, c'est-à-dire des vecteurs, de sorte que le coefficient effectif est bien un scalaire, de rang 0 . La symétrie du tenseur $\chi^{(2)}$ dépend de la symétrie d'orientation du milieu [2].

Dans le champ d'approximation qui a été choisi, les deux seules grandeurs qui gouvernent le couplage non linéaire sont $\Delta k$ et les $\kappa_{i}: \Delta k$ dépend uniquement des propriétés optiques linéaires via les indices de réfraction; $\kappa_{i}$ est fonction non seulement des indices de réfraction mais également des propriétés optiques non linéaires de second ordre. Le rôle de ces deux paramètres est expliqué dans le paragraphe suivant où est intégré le système (1).

Le système régissant une interaction à quatre ondes est du même type que le système (1), avec un champ supplémentaire et donc une équation différentielle supplémentaire [ $[5]$. 


\subsection{Rendement de conversion de génération de second harmonique (SHG) : hors accord de phase, en accord de phase et en quasi-accord de phase}

L'intégration du système (1) conduit à l'amplitude complexe de chaque onde, les solutions générales étant des fonctions elliptiques de Jacobi [1]. L'intégration se simplifie dans les cas où la variation d'une ou de deux des trois amplitudes est négligeable au cours de la propagation. Nous considérons ici une telle situation, en prenant l'exemple de la génération de second harmonique dans l'approximation de la pompe non dépeuplée, soit $\frac{\partial E_{1}^{\omega}}{\partial Z}=\frac{\partial E_{2}^{\omega}}{\partial Z}=0$. L'autre condition aux limites est : $E_{3}^{2 \omega}(X, Y, 0)=0$. L'intégration en $Z$ du système (1) est alors immédiate, ce qui permet ensuite de calculer la puissance générée à $2 \omega$ en intégrant $E_{3}^{2 \omega}(Z)$ sur la section transverse $(X, Y)$. Pour des faisceaux d'ondes planes à profil transverse gaussien, soit $E_{i}(X, Y, Z)=E_{0 i}(Z) \exp \left(-\frac{X^{2}+Y^{2}}{w_{i}{ }^{2}(Z)}\right)$ où $w_{i}(Z)$ est le rayon à $1 / \mathrm{e}$ de $E_{0 i}(Z)$, la puissance est calculée à partir de $P_{i}(L)=n_{i} \sqrt{\frac{\varepsilon_{0}}{\mu_{0}}} \frac{\pi}{4} w_{o}^{2}\left|E_{0 i}(L)\right|^{2}$, ce qui donne pour la puissance en Watt générée à $2 \omega$ :

$$
P^{2 \omega}(L)=B P_{1}^{\omega}(0) P_{2}^{\omega}(0) \frac{L^{2}}{w_{o}{ }^{2}} \operatorname{sinc}^{2}\left(\frac{\Delta k \cdot L}{2}\right)
$$

avec

$$
B=9462 \frac{2 N-1}{N} \frac{\chi_{\text {eff }}{ }^{2} T_{3}^{2 \omega} T_{1}^{\omega} T_{2}^{\omega}}{\lambda_{\omega}{ }^{2} n_{3}^{2 \omega} n_{1}^{\omega} n_{2}^{\omega}}
$$

- $P_{1}^{\omega}(0)$ et $P_{2}^{\omega}(0)$ sont les puissances incidentes correspondantes aux deux modes de polarisation, différents dans le cas général, soit $\mathbf{e}_{1}^{\omega} \neq \mathbf{e}_{2}^{\omega}$.

- Nous avons considéré que les deux faisceaux à $\omega$ ont un rayon identique $w_{o}[\mathrm{~m}]$.

- Les coefficients de transmission de Fresnel sont donnés par $T_{i}=\frac{4 n_{i}}{\left(1+n_{i}\right)^{2}}$.

- $L[\mathrm{~m}]$ est la longueur du cristal selon la direction de propagation.

- $N$ est le nombre de modes longitudinaux des faisceaux à $\omega$.

$-\lambda_{\omega}[\mathrm{m}]$ est la longueur d'onde fondamentale.

- $\chi_{\text {eff }}\left[\mathrm{m} . \mathrm{V}^{-1}\right]$ et $\Delta k\left[\mathrm{~m}^{-1}\right]$ sont les deux paramètres qui ont été définis au paragraphe précédent.

Notons que l'intégration du système (1) est indépendante du temps car la dispersion des vitesses de groupe a été négligée. Ainsi l'expression (2) correspond à la puissance instantanée $P^{2 \omega}(L, t)$ dans le cas de faisceaux impulsionnels.

Le rendement de génération de second harmonique est donné par :

$$
\eta_{S H G}=\frac{P^{2 \omega}(L)}{P_{1}^{\omega}(0)+P_{2}^{\omega}(0)}
$$


Généralement, la puissance incidente à $\omega, P_{t o t}^{\omega}=P_{1}^{\omega}(0)+P_{2}^{\omega}(0)$, est répartie de façon équivalente sur les deux modes de polarisation, soit $P_{1}^{\omega}(0)=P_{2}^{\omega}(0)=\frac{P_{t o t}^{\omega}}{2}$, le rendement de conversion, $\frac{P^{2 \omega}(L)}{P_{\text {tot }}^{\omega}}$, est alors proportionnel à la densité de puissance à $\omega$.

L'équation (2) montre que la puissance générée est proportionnelle à $\chi_{\text {eff }}{ }^{2}$, le coefficient effectif dépendant non seulement de la susceptibilité électrique de second ordre $\chi^{(2)}$, mais également de la configuration de polarisation $\left(\mathbf{e}_{1}^{\omega}, \mathbf{e}_{2}^{\omega}, \mathbf{e}_{3}^{2 \omega}\right)$. L'efficacité de l'interaction non linéaire est donc d'autant plus importante que la non-linéarité est forte, mais également que la configuration de polarisation est favorable, c'est-à-dire permet d'exprimer au mieux la non-linéarité du milieu.

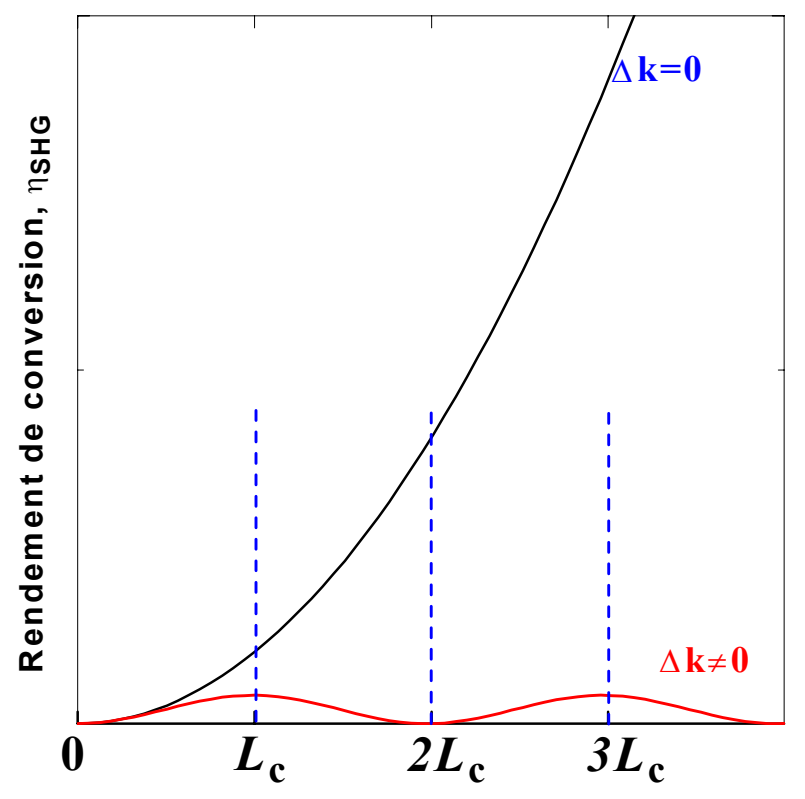

FIG. 2. Évolution du rendement de SHG en fonction de la distance d'interaction dans deux cas : hors accord de phase $(\Delta k \neq 0)$ et en accord de phase $(\Delta k=0) . L_{\mathrm{c}}$ est la longueur de cohérence de la SHG considérée.

Le paramètre $\Delta k$ conditionne la dépendance longitudinale du rendement de conversion $\eta_{S H G}$ comme le montre l'équation (2) et les courbes de la figure $\underline{2}$.

- à $\Delta k \neq 0, \eta_{S H G}$ varie comme $\sin ^{2}(\Delta k . L / 2)$; ainsi l'interférence entre la polarisation non linéaire, $\mathbf{P}^{N L}(L)=\varepsilon_{0}\left(\chi^{(2)}(2 \omega): \mathbf{e}_{1}^{\omega} \otimes \mathbf{e}_{2}^{\omega}\right) E_{1}^{\omega}(L) E_{2}^{\omega}(L) \exp \left(j\left(k_{1}^{\omega}+k_{2}^{\omega}\right) L\right)$, et le champ rayonné, $\mathbf{E}_{3}^{2 \omega}(L)=\mathbf{e}_{3}^{2 \omega} E_{3}^{2 \omega}(L) \exp \left(j k_{3}^{2 \omega} L\right)$, est alternativement constructive et destructive avec une période égale à $2 L_{c}$ : après une propagation sur la distance $L_{c}$, appelée longueur de cohérence de l'interaction, le déphasage a changé de $\pi$, soit $L_{c}=\frac{\pi c}{\omega\left[2 n_{3}^{2 \omega}-\left(n_{1}^{\omega}+n_{2}^{\omega}\right)\right]}$. La dispersion en longueur d'onde des indices de réfraction des milieux classiquement utilisés pour la conversion de fréquence est telle que $L_{c}$ a des valeurs comprises entre $1 \mu \mathrm{m}$ dans l'ultraviolet et $100 \mu \mathrm{m}$ dans l'infrarouge moyen. Cette situation, appelée « hors accord de phase », conduit donc à des rendements de conversion extrêmement faibles, de sorte que l'approximation de la pompe non dépeuplée est valide. Ce cas n'est donc absolument pas 
intéressant pour des applications commerciales, mais néanmoins nous verrons au §5.2.1 qu'il peut être utile pour l'étude du tenseur $\chi^{(2)}$.

- à $\Delta k=0$, la polarisation non linéaire et le champ rayonné restent en phase tout au long de la propagation dans le cristal : l'interférence est alors constructive et la puissance de l'onde rayonnée varie en $L^{2}$. Ainsi, il y a transfert d'énergie de la polarisation non linéaire au champ rayonné tout au long de la propagation. Dans ce cas, appelé « accord de phase », le rendement de conversion peut atteindre des valeurs importantes, de sorte que l'approximation de la pompe non dépeuplée, qui a été stipulée pour les calculs précédents, n'est plus valable. Audelà de $5 \%$ de rendement de conversion en énergie, la dépendance longitudinale de la puissance rayonnée en accord de phase doit être décrite par la fonction hyperbolique $\tanh ^{2}\left(C I_{\text {tot }}^{\omega} L\right)$ où $C$ est une constante dépendant de la direction de propagation considérée et $I_{t o t}^{\omega}$ la densité de puissance de l'onde fondamentale : des rendements en énergie de génération de second harmonique de l'ordre de $80 \%$ peuvent être atteints dans des cristaux de $\mathrm{KH}_{2} \mathrm{PO}_{4}(\mathrm{KDP})$ ou $\mathrm{KTiOPO}_{4}(\mathrm{KTP})$.

Il est également intéressant de considérer l'effet d'une variation de $\Delta k$ autour de 0 , à longueur d'interaction $L$ constante, due à la variation d'une grandeur de dispersion $\xi$ des indices de réfraction, $n(\xi)$ : par exemple l'angle que fait la direction de propagation par rapport au repère optique dans le cas des milieux anisotropes, la longueur d'onde, la température ou un champ électrique appliqué. La variation de la puissance rayonnée est alors proportionnelle à $\operatorname{sinc}^{2}\left(\frac{\Delta k(\xi) \cdot L}{2}\right)$ comme le montre la figure 3 . Notons que l'allure de la courbe est symétrique par rapport à la valeur $\xi_{\mathrm{AP}}$ uniquement dans le cas où $\Delta k$ est proportionnel à $\xi$. Les quantités qui caractérisent les effets d'un écart à la valeur $\xi_{\mathrm{AP}}$ sont habituellement définies comme la variation de la grandeur considérée, $\delta \xi$, conduisant à une variation du désaccord de phase $\Delta k(\delta \xi)$ de $\frac{2 \pi}{L}$. $\delta \xi$ est également la largeur du pic à 0,405 de la valeur maximale. $L \delta \xi$, qui est appelée acceptance ou tolérance, est donc une caractéristique de la direction de propagation considérée. Ainsi, plus $L$ est petite et plus les tolérances, angulaires, spectrale, thermique..., sont grandes, ce qui est favorable vis-à-vis de la divergence et de la largeur spectrale du laser de pompe ou des fluctuations thermiques respectivement. Les valeurs de tolérances sont données dans le tableau III du $\S 7$ pour les principaux cristaux non linéaires. Lorsque l'interaction non linéaire est réalisée avec des cristaux à faces planes, ce qui est généralement le cas, l'expression (2) à $\Delta k \neq 0$ sera d'autant moins valide que l'angle entre le faisceau incident et la normale à la face d'entrée est grand : en effet il faut tenir compte du fait que les ondes réfractées à $\omega$ et $2 \omega$ ne sont pas colinéaires lorsque la SHG hors accord de phase est réalisée en incidence oblique [6]].

Une autre alternative permettant d'obtenir un rendement de conversion important est le quasiaccord de phase : il est réalisé dans des milieux artificiels où le signe du coefficient effectif a été périodiquement alterné, la période d'inversion correspondant exactement à la longueur de cohérence $L_{c}$ de l'interaction considérée. Cette inversion du signe permet un déphasage "artificiel» de $\pi$ qui, venant s'ajouter au déphasage «naturel» dû à la dispersion en longueur d'onde des indices de réfraction, permet une remise en phase de la polarisation non linéaire avec le champ rayonné. Ainsi, la puissance à $2 \omega$ qui est générée sur une longueur de cohérence n'est pas détruite sur la longueur suivante, ce qui permet de garder une interférence constructive tout au long de la propagation comme le montre la figure $\underline{4}$. Le rendement de conversion ainsi obtenu est alors $\frac{4}{\pi^{2}}$ fois le rendement qui serait obtenu en accord de phase [7]. L'inversion du signe du coefficient effectif est classiquement réalisé par inversion des domaines ferro-électriques après cristallogenèse, dans $\mathrm{LiNbO}_{3}$ ou KTP par exemple, ou par croissance alternée dans le cas de GaAs. 


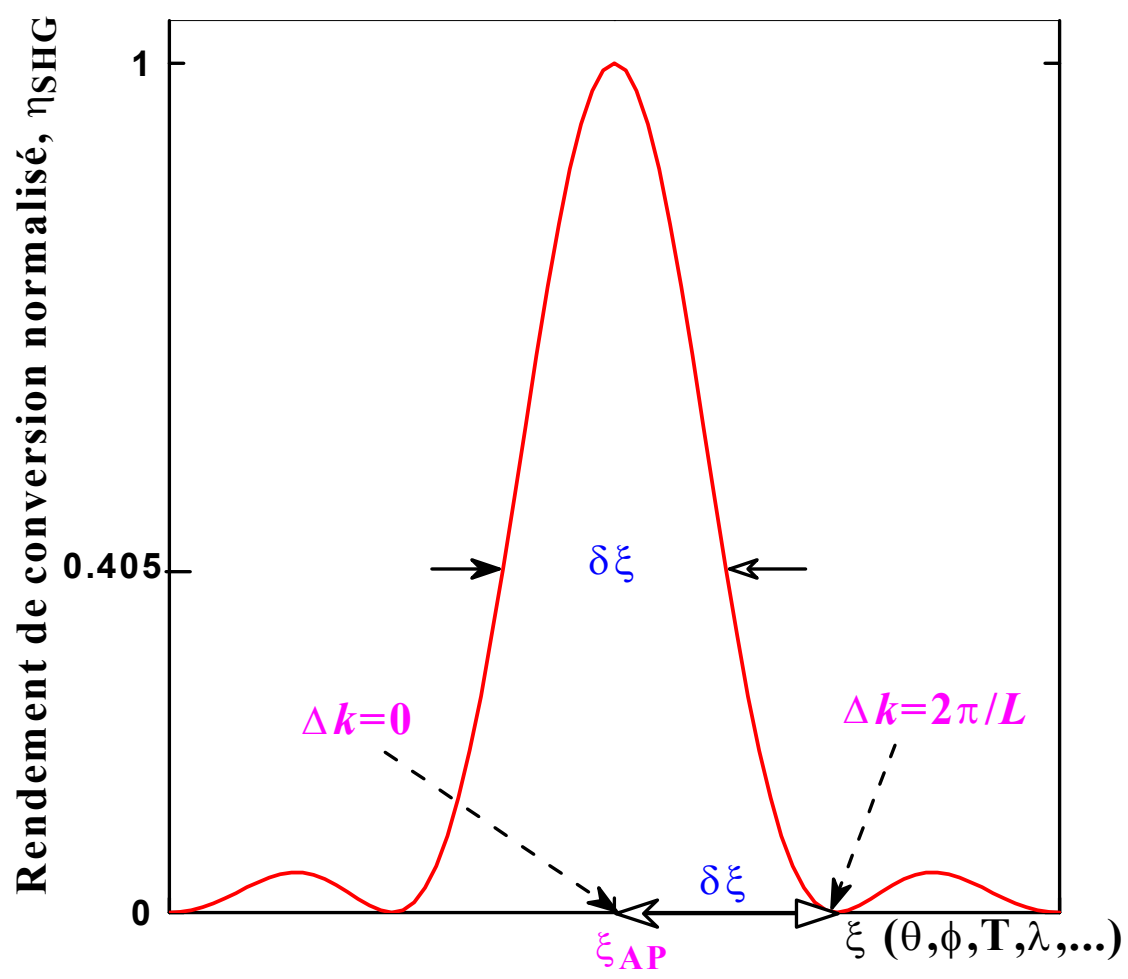

FIG. 3. Variation du rendement de SHG en fonction du paramètre $\xi$ dont dépendent les indices de réfraction : $(\theta, \phi)$ coordonnées sphériques de la direction de propagation, $T$ température, $\lambda$ longueur d'onde. $L$ est la longueur d'interaction.

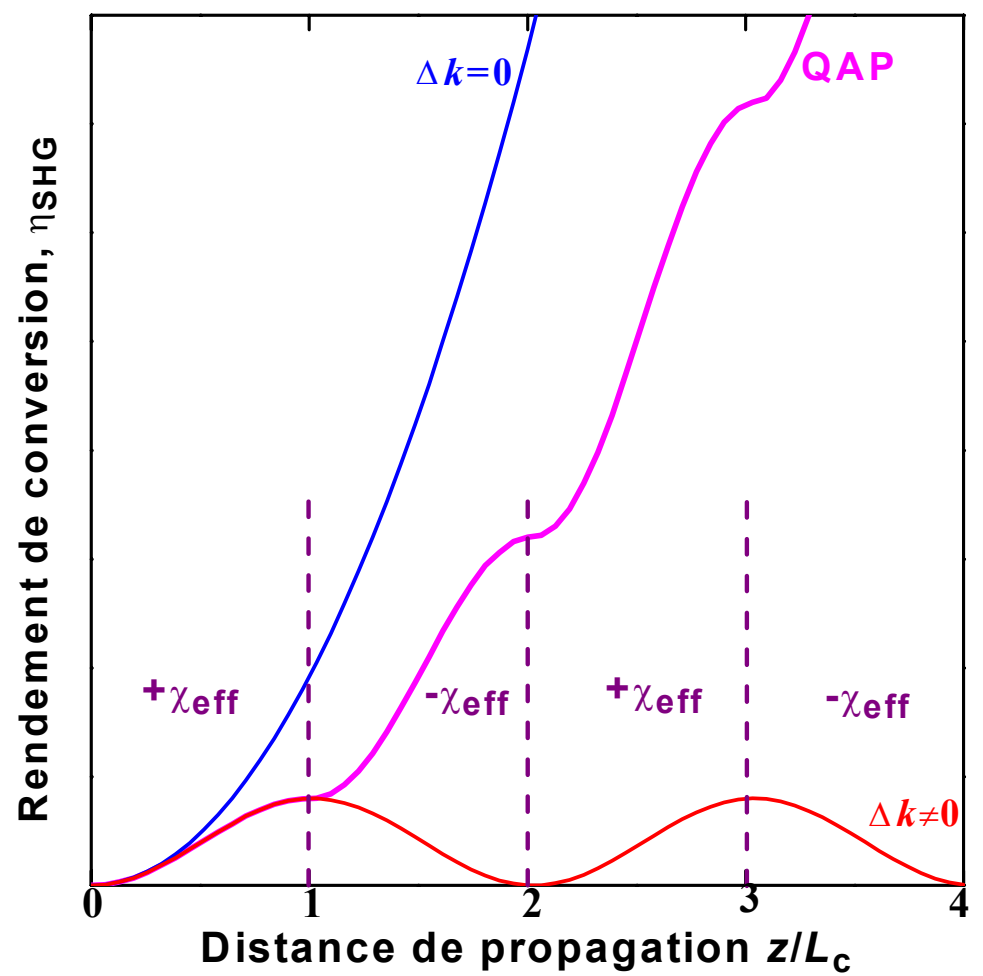

FIG. 4. Comparaison entre les rendements de SHG obtenus en quasi-accord de phase (QAP), accord de phase $(\Delta k=0)$ et hors accord de phase $(\Delta k \neq 0) . L_{c}$ est la longueur de cohérence définie par $L_{\mathrm{c}}=\pi / \Delta k$. 


\subsection{Prise en compte de la double réfraction et de la dispersion des vitesses de groupe}

Nous verrons dans le $\S 4.1$. que les interactions de conversion de fréquence en accord de phase sont réalisées dans des milieux anisotropes. Il faut alors tenir compte, dans le cas général, de la double réfraction : elle a pour effet une séparation spatiale des faisceaux en interaction, ce qui conduit à une atténuation de l'efficacité du couplage. Cette atténuation est d'autant plus forte que les faisceaux sont de petite taille. D'autre part en régime impulsionnel, la dispersion des vitesses de groupe peut conduire à une séparation temporelle des impulsions lorsque celles-ci sont de courtes durées, que le milieu soit isotrope ou anisotrope. La prise en compte de ces deux phénomènes nécessite de remplacer dans le système (1) l'opérateur $\frac{\partial}{\partial Z}$ par l'opérateur $\frac{\partial}{\partial Z}+\tan \rho_{i_{X}} \frac{\partial}{\partial X}+\tan \rho_{i_{Y}} \frac{\partial}{\partial Y}+\frac{1}{v_{g_{i}}} \frac{\partial}{\partial t}: \rho_{i_{X}}$ et $\rho_{i_{Y}}$ sont les angles de double réfraction de l'onde $i$ dans les plans $(X, Z)$ et $(Y, Z) ; v_{g_{i}}=\left(\frac{\partial \omega}{\partial k}\right)_{\omega=\omega_{i}}$ est la vitesse de groupe de l'onde $i$. L'intégration du système d'équations couplées avec ces nouveaux opérateurs différentiels conduit à l'expression (2) multipliée par deux fonctions $G\left(L, w_{o}, \rho_{i_{X}}, \rho_{i_{Y}}\right)$ et $G\left(L, \tau_{i}, v_{g_{i}}\right)$ qui sont relatives respectivement à l'atténuation due à la double réfraction et celle due à la dispersion des vitesses de groupe, $\tau_{\mathrm{i}}$ étant la durée d'impulsion de l'onde $i$. Dans le cas d'un cristal de longueur $L=1 \mathrm{~cm}$, ces deux effets sont négligeables, c'est-à-dire que les deux fonctions tendent vers 1 , pour $w_{\mathrm{o}} \geq 1 \mathrm{~mm}$ et $\tau_{\mathrm{i}} \geq 100 \mathrm{ps}$. Notons que les angles de double réfraction et les vitesses de groupe dépendent uniquement des indices de réfraction.

Les calculs qui ont été développés dans ce paragraphe constituent un cadre tout à fait adapté aux situations dans lesquelles sont mesurées les propriétés optiques non linéaires: en effet, la génération de second harmonique est souvent considérée et les rendements de conversion associés sont souvent faibles.

\subsection{Bilan des paramètres optiques régissant les interactions de somme et différence de fréquences}

Des calculs analogues menés dans les cas de la somme de fréquences, $\omega_{1}+\omega_{2}=\omega_{3}$, et de la différence de fréquences, $\omega_{1}-\omega_{2}=\omega_{3}$, avec $\omega_{1} \neq \omega_{2}$, conduisent à l'identification des mêmes paramètres que ceux qui régissent la génération de second harmonique $[\underline{1}, \underline{2}, \underline{3}, \underline{4}]$. Le tableau $\underline{I}$, qui rassemble les cinq paramètres identifiés, montre l'importance considérable des propriétés optiques linéaires, d'autant plus qu'il faut préalablement à cette analyse considérer la fenêtre de transparence du milieu : celle-ci doit être la plus étendue possible, et dans tous les cas ouverte à des gammes de longueurs d'onde intéressantes pour les applications.

La connaissance de ces paramètres permet une évaluation complète des potentialités d'un milieu pour la conversion de fréquence. Les indices de réfraction mesurés avec une précision de l'ordre de $10^{-3}$ permettent de calculer de façon satisfaisante les fonctions d'atténuation dues à la double réfraction et à la dispersion des vitesses de groupe. Une telle précision sur les indices est accessible par la mesure du minimum de déviation ou de l'angle de réflexion totale. Par contre, le calcul des conditions d'accord de phase ou de quasi-accord de phase nécessite une précision sur l'indice qui est inaccessible expérimentalement dans beaucoup de cas, de sorte que, dans la pratique, il est absolument nécessaire de mesurer directement ces propriétés. La mesure est également nécessaire dans le cas du coefficient effectif : en effet, à ce jour, les meilleurs modèles de non-linéarité optique ne permettent pas de donner mieux que l'ordre de grandeur des différents éléments des tenseurs de susceptibilité électrique de second et troisième ordre [8]. 


\begin{tabular}{|c|c|c|c|c|}
\hline \multirow[t]{2}{*}{ Propriétés optiques } & linéaires & non linéaires & \multirow[b]{2}{*}{ Calcul } & \multirow[b]{2}{*}{ Mesure } \\
\hline & $n=\sqrt{\chi^{(1)}+1}$ & $\chi^{(2)}$ & & \\
\hline$\chi_{\text {eff }}$ & & & & \\
\hline $\begin{array}{c}\text { Conditions d'accord de } \\
\text { phase }\left(\theta_{A P}, \varphi_{A p}, \boldsymbol{T}_{A P}, \lambda_{A P}\right)\end{array}$ & & & & \\
\hline$G^{D R}\left(\rho_{i}\right)$ & & & & \\
\hline$G^{D V G}\left(v_{g}^{i}\right)$ & & & & \\
\hline $\begin{array}{c}\text { Conditions de quasi-accorc } \\
\text { de phase }\left(L_{c}\right)\end{array}$ & & & & \\
\hline
\end{tabular}

TAB. II. Principaux paramètres gouvernant les interactions de conversion de fréquences et dépendance vis-à-vis des propriétés optiques linéaires et non linéaires : le coefficient effectif, $\chi_{\text {eff }}$; les angles, température et longueurs d'onde d'accord de phase, $\theta_{A P}, \varphi_{A p}, T_{A P}, \lambda_{A P}$ respectivement; la fonction d'atténuation $G^{D R}$ due à la double réfraction $\rho_{i}$; la fonction d'atténuation $G^{D V G}$ due à la dispersion des vitesses de groupe $v_{g}^{i}$; la longueur de cohérence $L_{c}$.

Dans ce qui suit, nous nous intéressons donc aux deux grandeurs qui nécessitent une mesure, à savoir les directions d'accord de phase et le coefficient effectif. Les principales méthodes utilisées sont décrites et comparées entre elles. La mesure de la longueur de cohérence pour le quasi-accord de phase ne fait pas l'objet d'un paragraphe spécifique. Elle est néanmoins abordée dans le paragraphe consacré à l'étude du tenseur $\chi^{(2)}$ dont une méthode repose en partie sur la mesure de la longueur de cohérence.

\section{4. ÉTUDE DES PROPRIÉTÉS D’ACCORD DE PHASE}

\section{1. Éléments de base sur l'accord de phase}

La mise en phase de la polarisation non linéaire et du champ rayonné correspond d'un point de vue corpusculaire à la conservation de la quantité de mouvement des photons en interaction, $\hbar \mathbf{k}_{1}+\hbar \mathbf{k}_{2}=\hbar \mathbf{k}_{3}$, la conservation de l'énergie, $\hbar \omega_{1}+\hbar \omega_{2}=\hbar \omega_{3}$, étant toujours assurée. Dans le cas où les trois ondes associées se propagent avec des vecteurs d'onde colinéaires entre eux, la relation d'accord de phase devient :

$$
\omega_{1} n_{1}+\omega_{2} n_{2}=\omega_{3} n_{3}
$$

Dans l'hypothèse d'un milieu dont l'indice de réfraction ne dépendrait pas de la longueur d'onde, $n_{1}=n_{2}=n_{3}$, l'expression (3) serait équivalente à la relation de conservation de l'énergie, de sorte que l'accord de phase serait réalisé quelle que soit l'interaction de conversion de fréquence considérée. Cette situation est fictive, car tout milieu réel est dispersif.

La dispersion en longueur d'onde est bien décrite par un modèle d'oscillateurs électroniques et ioniques indépendants [9]. Dans ce cadre, pour des longueurs d'onde comprises entre deux oscillateurs de longueurs d'onde de résonance $\lambda_{c}$ et $\lambda_{e}$, l'indice de réfraction est donné par :

$$
n^{2}(\lambda)=a+\frac{b \lambda^{2}}{\lambda^{2}-c}+\frac{d \lambda^{2}}{\lambda^{2}-e}
$$


Les longueurs d'onde de résonance des deux pôles sont $\lambda_{c}=\sqrt{c}$ et $\lambda_{e}=\sqrt{e}$. Pour obtenir une description plus précise de la dispersion en longueur d'onde des indices principaux, en particulier pour les cristaux ayant une fenêtre de transparence large, il est utile de considérer d'autres types d'équations, faisant intervenir plus de paramètres. Une solution consiste à ajouter un ou deux oscillateurs à l'équation (4). Une autre forme peut aussi être utilisée, soit :

$$
n^{2}(\lambda)=a+\frac{b}{1-\left(\frac{c}{\lambda}\right)^{p}}+\frac{d}{1-\left(\frac{e}{\lambda}\right)^{q}}
$$

Les expressions ( $\underline{4})$ et ( $\underline{5})$ montrent que, dans le domaine de transparence du milieu, l'indice de réfraction est une fonction décroissante de la longueur d'onde, ce qui est qualifié de "dispersion normale ».

Considérons dans un premier temps un milieu réel isotrope. Dans ce cas, les trois indices principaux de réfraction sont égaux, soit : $n_{x}=n_{y}=n_{z}(=n)$. Le repère $(x, y, z)$, appelé " repère optique », est le repère dans lequel le tenseur des indices de réfraction est diagonal. Ce repère ne doit pas être confondu avec le repère $(X, Y, Z)$, repère du laboratoire défini précédemment, dans lequel sont écrites et intégrées les équations de propagation. Dans un milieu isotrope la relation d'accord de phase (3) se réduit pour la génération de second harmonique à $n^{\omega}=n^{2 \omega}$. Cette égalité ne peut évidemment pas être vérifiée si le milieu est dispersif car il y a inégalité stricte entre les deux indices de réfraction comme le montre la figure $\underline{5}$.
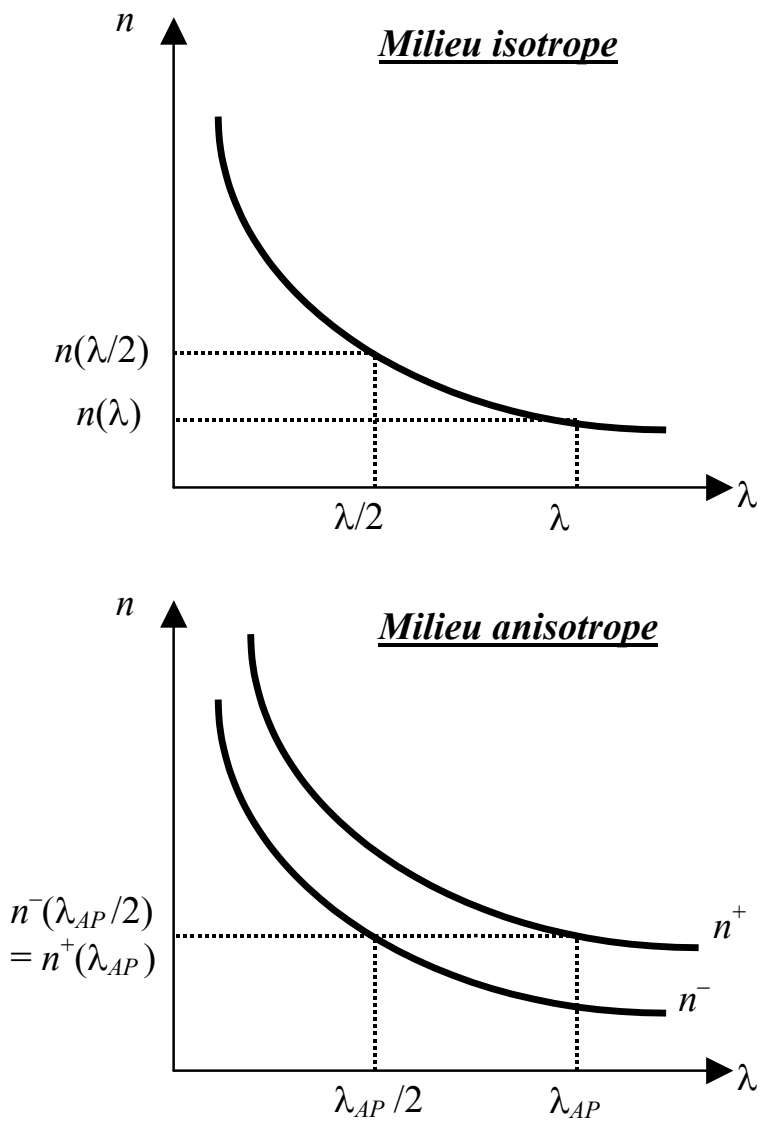

FIG. 5. Situation des milieux isotropes et anisotropes vis-à-vis de la compensation de la dispersion en longueur d'onde de l'indice de réfraction $n(\lambda)$ en vue de réaliser l'accord de phase pour la SHG de type I : impossibilité pour les milieux isotropes; existence possible d'une SHG en accord de phase pour la longueur d'onde fondamentale $\lambda_{A P}$ dans les milieux anisotropes. Les indices $n^{-}$et $n^{+}$sont les solutions de l'équation de Fresnel relatives à la direction d'accord de phase de coordonnées sphériques $\left(\theta_{A P}, \phi_{A P}\right)$. 
L'utilisation d'un cristal anisotrope est l'alternative classiquement utilisée pour la réalisation de l'accord de phase. En effet, la biréfringence permet de compenser la dispersion en longueur d'onde comme le montre la figure $\underline{5}$ avec l'exemple simple de la génération de second harmonique où les deux ondes fondamentales ont le même mode de polarisation: $\mathbf{e}_{1}^{\omega}=\mathbf{e}_{2}^{\omega}$. Dans ce cas, la relation d'accord de phase est $n^{\omega}=n^{2 \omega}$. La figure $\underline{5}$ montre bien que dans la direction de propagation considérée, de coordonnées sphériques $\left(\theta_{A P}, \phi_{A P}\right)$ dans le repère optique, l'égalité précédente peut être vérifiée pour une longueur d'onde fondamentale particulière $\lambda_{A P}^{\omega}$ à condition que l'onde fondamentale soit polarisée dans le mode + et l'onde harmonique dans le mode - , soit : $n_{\omega}^{+}\left(\theta_{A P}, \phi_{A P}\right)=n_{2 \omega}^{-}\left(\theta_{A P}, \phi_{A P}\right)$. Les indices $n^{+}\left(\theta_{A P}, \phi_{A P}\right)$ et $n^{-}\left(\theta_{A P}, \phi_{A P}\right)$ sont les deux solutions de l'équation de Fresnel dans la direction de propagation $\left(\theta_{A P}, \phi_{A P}\right)$ [3] dont les valeurs sont données par :

$$
n^{ \pm}=\left(\frac{2}{-B \pm\left(B^{2}-4 C\right)^{1 / 2}}\right)^{1 / 2}
$$

avec

$$
\begin{aligned}
& B=-u_{x}{ }^{2}(b+c)-u_{y}{ }^{2}(a+c)-u_{z}{ }^{2}(a+b), C=b c u_{x}{ }^{2}+a c u_{y}{ }^{2}+a b u_{z}{ }^{2}, \\
& a=n_{x}{ }^{-2}(\omega), b=n_{y}{ }^{-2}(\omega), c=n_{z}{ }^{-2}(\omega),
\end{aligned}
$$

où $\left(u_{x}, u_{y}, u_{z}\right)$ sont les coordonnées cartésiennes du vecteur unitaire colinéaire au vecteur d'onde reliées aux coordonnées sphériques par $u_{x}=\cos \phi_{A P} \sin \theta_{A P}, u_{y}=\sin \phi_{A P} \sin \theta_{A P}$ et $u_{z}=\cos \theta_{A P}$. Les solutions $n^{ \pm}(\theta, \phi)$ forment une surface à deux nappes appelée «surface des indices ». Rappelons qu'il y a deux classes de milieux anisotropes: la classe optique uniaxe, définie par $n_{x}=n_{y}\left(=n_{o}\right.$ indice ordinaire) $\neq n_{z}$ ( $=n_{e}$ indice extraordinaire) et la classe biaxe, $n_{x} \neq n_{y} \neq n_{z}$. La surface des indices d'un cristal uniaxe est formée d'une nappe sphérique de rayon $n_{o}$ et d'une nappe ellipsoïdale d'axes $n_{o}$ et $n_{e} ;$ un point courant de l'ellipsoïde est alors donné par :

$$
n_{e}(\theta, \phi)=\left(\frac{\cos ^{2} \theta}{n_{o}{ }^{2}}+\frac{\sin ^{2} \theta}{n_{e}{ }^{2}}\right)^{-1 / 2}
$$

Cette relation est valable quel que soit $\phi$ car $O z$ est un axe de symétrie $A \infty$ pour la surface des indices d'un uniaxe. D'autre part, la surface des indices présente un seul ombilic, par lequel passe $O z$ qui est appelé axe optique. La surface des indices est plus compliquée dans le cas des biaxes pour lesquels les nappes ne présentent des sections circulaires ou elliptiques que dans les plans principaux $(x, y),(x, z)$ et $(y, z)[\underline{3}]$. Il y a deux ombilics pour les biaxes, soit deux axes optiques qui sont symétriques par rapport à $O z$ et situés dans le plan $(x, z)$ par convention. D'un point de vue général, la relation d'accord de phase colinéaire des interactions paramétriques à trois ondes peut être vérifiée pour trois triplets d'indices dans les milieux anisotropes présentant une dispersion normale :

Type I $\quad \omega_{3} n^{-}\left(\omega_{3}, \theta, \phi\right)=\omega_{1} n^{+}\left(\omega_{1}, \theta, \phi\right)+\omega_{2} n^{+}\left(\omega_{2}, \theta, \phi\right)$

Type II

$$
\omega_{3} n^{-}\left(\omega_{3}, \theta, \phi\right)=\omega_{1} n^{-}\left(\omega_{1}, \theta, \phi\right)+\omega_{2} n^{+}\left(\omega_{2}, \theta, \phi\right)
$$

Type III

$$
\omega_{3} n^{-}\left(\omega_{3}, \theta, \phi\right)=\omega_{1} n^{+}\left(\omega_{1}, \theta, \phi\right)+\omega_{2} n^{-}\left(\omega_{2}, \theta, \phi\right)
$$

$\grave{A}$ ces trois situations correspondent trois configurations de polarisation : $\left(\mathbf{e}_{1}^{+}, \mathbf{e}_{2}^{+}, \mathbf{e}_{3}^{-}\right)$pour le type I, $\left(\mathbf{e}_{1}^{-}, \mathbf{e}_{2}^{+}, \mathbf{e}_{3}^{-}\right)$pour le type II, $\left(\mathbf{e}_{1}^{+}, \mathbf{e}_{2}^{-}, \mathbf{e}_{3}^{-}\right)$pour le type III. Les types II et III sont évidemment équivalents dans le cas de la génération de second harmonique.

Pour une interaction donnée, caractérisée par les pulsations $\omega_{1}, \omega_{2}, \omega_{3}$ et le type, I, II ou III, l'accord de phase est vérifié pour un ensemble de couples $\left(\theta_{A P}, \phi_{A P}\right)$. Ces couples se calculent aisément 
à partir de la relation d'accord de phase considérée et de l'expression des solutions de l'équation de Fresnel. Il existe des expressions analytiques pour les angles d'accord de phase dans tous les cas où deux des trois indices de réfraction des ondes en interaction sont relatifs à une nappe de section circulaire, soit : pour les interactions entre deux ondes ordinaires et une onde extraordinaire dans les uniaxes et dans les plans principaux $(x, z)$ et $(y, z)$ des biaxes; pour les interactions couplant deux ondes extraordinaires à une onde ordinaire dans le plan principal $(x, y)$ des biaxes. Dans tous les autres cas il est nécessaire de faire une résolution numérique. Considérons un exemple de solution analytique avec la génération de second harmonique de type I dans un cristal uniaxe tel que $n_{e}<n_{o}$ (uniaxe négatif). La relation d'accord de phase s'écrit alors $n_{e}^{2 \omega}\left(\theta_{A P}^{I}, \phi\right)=n_{o}^{\omega}$ quel que soit $\phi$. L'angle $\theta_{A P}^{I}$ est alors donné par l'intersection d'un cercle et d'une ellipse comme le montre la figure $\underline{6}$; il a pour expression :

$$
\theta_{A P}^{I}=\arcsin \left(\left[\frac{n_{o}^{-2}(\omega)-n_{o}^{-2}(2 \omega)}{n_{e}^{-2}(2 \omega)-n_{o}^{-2}(2 \omega)}\right]^{1 / 2}\right)
$$

Il existe donc un ensemble de directions d'accord de phase formant un cône de révolution autour de $O z$ d'ouverture $\theta_{A P}^{I}$. Compte tenu de la symétrie de la surface des indices des uniaxes, les directions d'accord de phase d'une interaction donnée forment toujours deux cônes, symétriques par rapport au plan $x-y$ et dont l'axe de révolution est $O z$, comme le montre la figure $\underline{7}$. Pour les biaxes la situation est beaucoup plus compliquée : les surfaces formées par les directions d'accord de phase sont des cônes déformés avec cinq enroulements possibles qui sont décrits figure $\underline{8}$ dans un seul octant de l'espace [10].

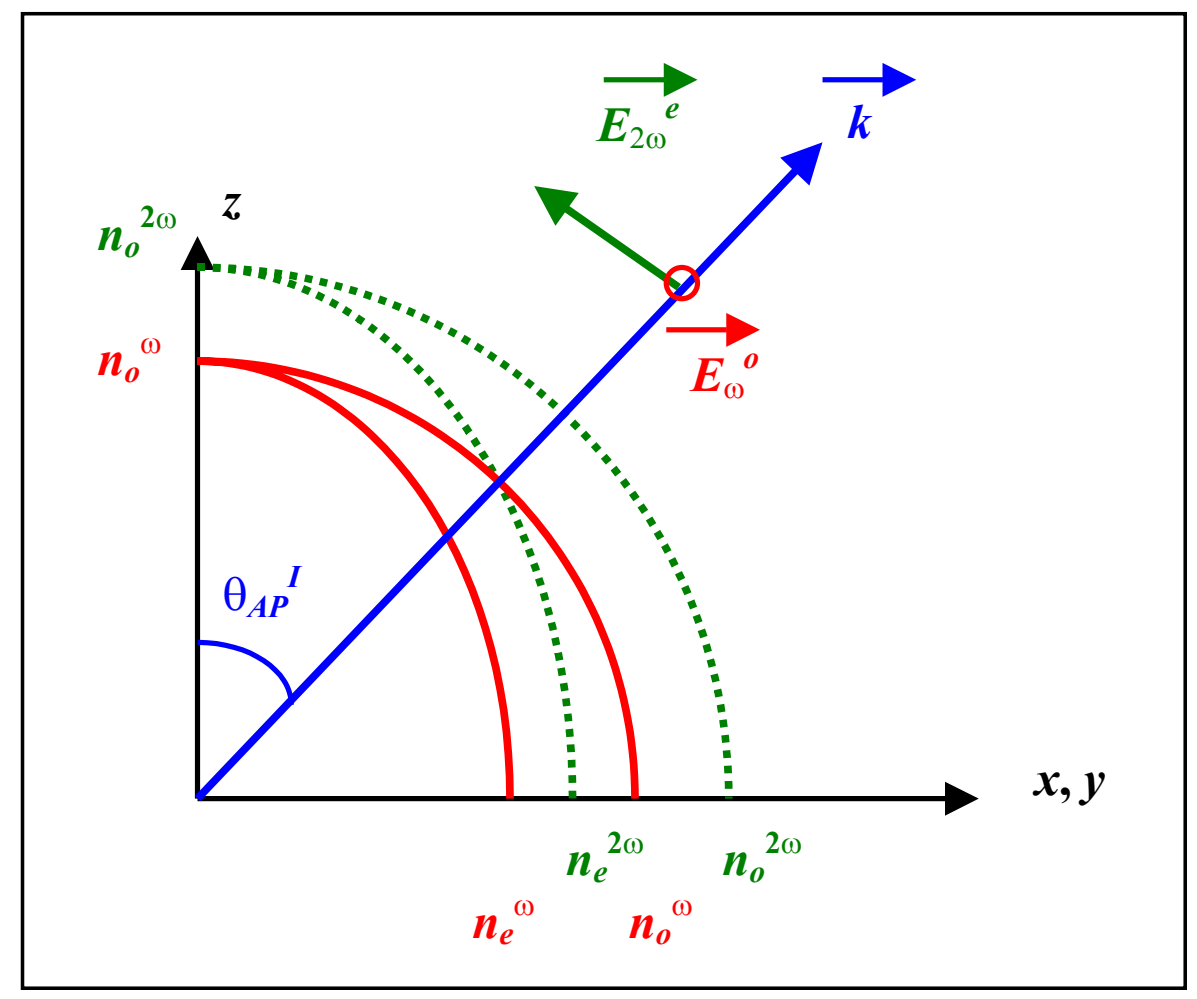

FIG 6. Section de la surface des indices à $\omega$ et $2 \omega$ dans un plan contenant l'axe optique $(O z)$. Les $n_{o, e}^{\omega, 2 \omega}$ sont les indices de réfraction principaux ordinaires $(o)$ et extraordinaires $(e) . \theta_{A P}^{I}$ est l'angle d'accord de phase de la SHG de type I dont le vecteur d'onde est $\vec{k}$ et la configuration de polarisation $\left(\vec{E}_{\omega}^{o}, \vec{E}_{2 \omega}^{e}\right)$. $(x, y, z)$ est le repère optique. 


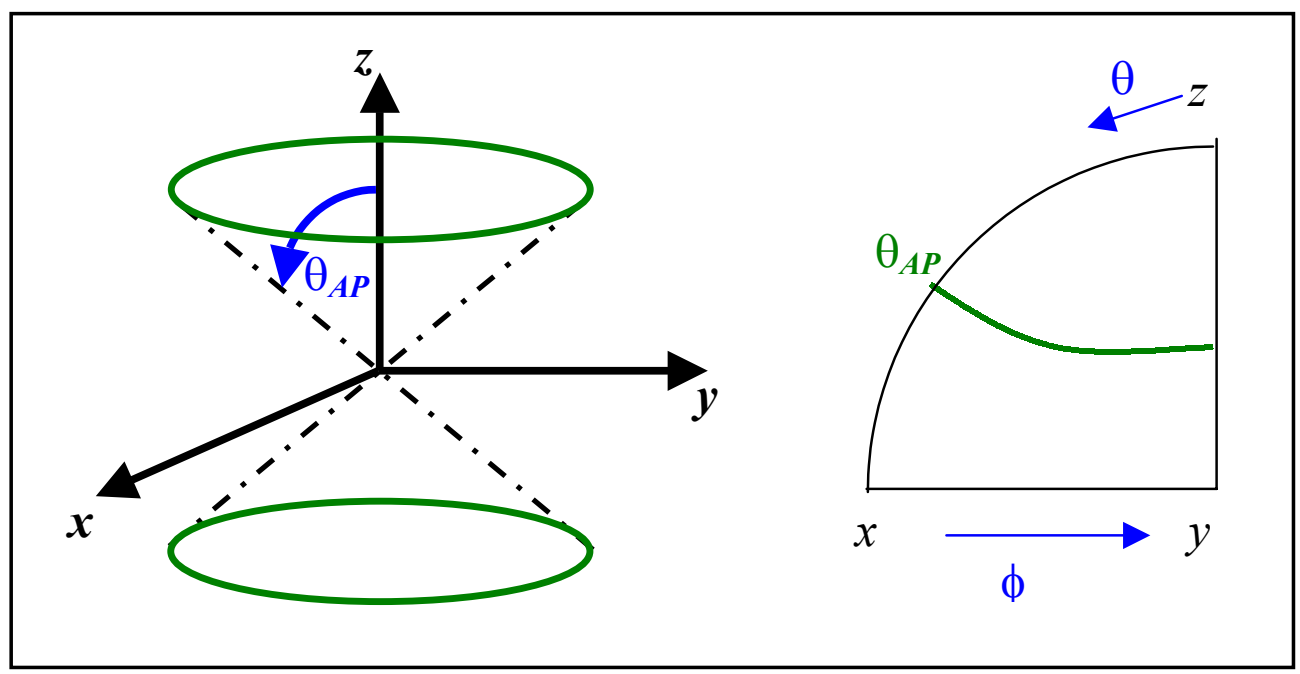

FIG 7. Cône des directions d'accord de phase d'ouverture $\theta_{A P}$ dans le cas des cristaux uniaxes. $(x, y, z)$ est le repère optique.

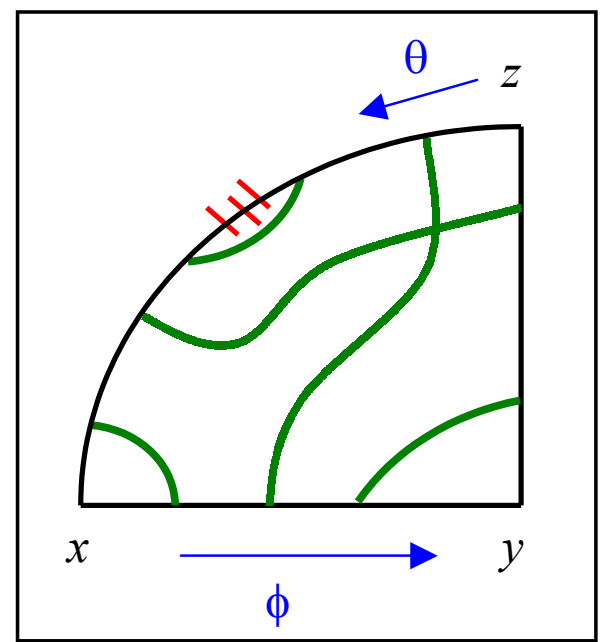

FIG 8. Types de localisation des directions d'accord de phase dans le cas des cristaux biaxes. Les trois traits coupant le plan $x-z$ indiquent la localisation des axes optiques de réfraction conique intérieure relatifs aux trois ondes couplées de pulsations $\omega_{1}, \omega_{2}, \omega_{3}$.

\subsection{Détermination des directions d'accord de phase}

\subsubsection{Méthode indirecte}

C'est la méthode classiquement utilisée : les directions d'accord de phase sont calculées à partir des indices de réfraction mesurés préalablement.

\subsubsection{Description de la méthode}

Les données de base pour le calcul sont les équations de dispersion en longueur d'onde des indices de réfraction. Elles sont obtenues à partir des indices de réfraction mesurés à plusieurs longueurs d'onde. La méthode de mesure la plus couramment utilisée est celle du prisme au minimum de déviation qui est décrite ci-après. 
Pour les cristaux uniaxes il suffit d'un seul prisme dont la bissectrice est orientée selon l'axe optique. Au minimum de déviation, les ondes se propagent orthogonalement à la bissectrice, c'est-àdire dans le plan $x-y$ du repère optique, qu'il s'agisse du mode ordinaire ou extraordinaire, comme l'indique la figure $\underline{9}$. La mesure des angles au minimum de déviation $D_{e}^{m}$ et $D_{o}^{m}$ dans les deux configurations de polarisation possibles, extraordinaire et ordinaire respectivement, conduit aux deux indices principaux $n_{e}$ et $n_{o}$, soit pour un prisme d'angle $\Delta$ :

$$
n_{e, o}=\frac{\sin \left(\frac{\Delta+D_{e, o}^{m}}{2}\right)}{\sin \left(\frac{\Delta}{2}\right)}
$$

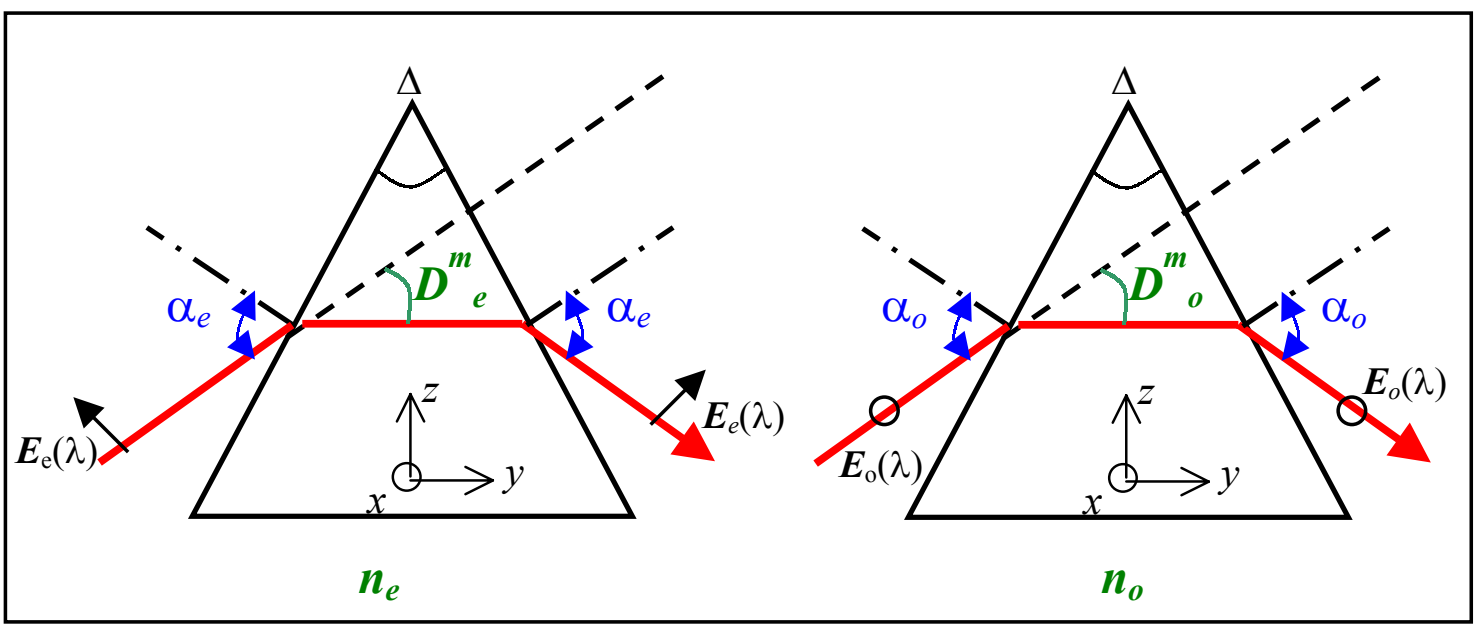

FIG 9. Configuration des champs au minimum de déviation d'un prisme en cristal uniaxe permettant la détermination des deux indices principaux de réfraction $n_{o}$ et $n_{e}$. $(x, y, z)$ est le repère optique. $\boldsymbol{E}_{o, e}(\lambda)$ sont les champs électriques des ondes ordinaire et extraordinaire à la longueur d'onde $\lambda . \alpha_{o, e}$ et $D_{o, e}^{m}$ sont les angles de réfraction au minimum de déviation.

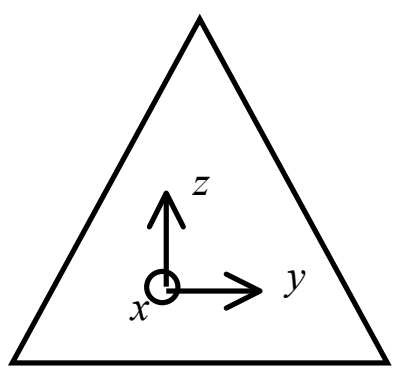

$n_{x}, n_{z}$

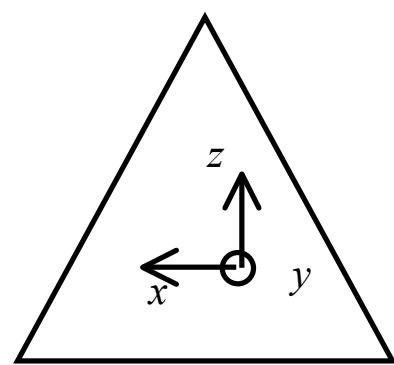

$n_{y}, n_{z}$

FIG. 10. Orientation possible des deux prismes nécessaires à la mesure des trois indices de réfraction principaux $n_{x}, n_{y}$ et $n_{z}$ d'un cristal biaxe. $(x, y, z)$ est le repère optique. 
Pour les cristaux biaxes il faut deux prismes orientés différemment. Six possibilités existent dont les deux données en exemple figure 10. Les deux prismes ont l'axe $O z$ comme bissectrice mais les deux plans d'incidence sont orientés différemment : un contient l'axe $O x$ et l'autre, l'axe $O y$. Ainsi il est possible de déterminer $n_{x}$ et $n_{z}$ avec le premier prisme en polarisant le rayonnement selon $O x$ et $O z$ respectivement ; de même le deuxième permet la détermination de $n_{y}$ et $n_{z}$ en polarisant selon $O y$ et $O z$ respectivement. L'indice $n_{z}$ est déterminé deux fois dans cet exemple, ce qui n'est pas inutile car cela permet de vérifier la fiabilité des mesures.

L'interpolation des indices de réfraction principaux mesurés à plusieurs longueurs d'onde permet l'établissement des équations de dispersion en longueur d'onde. L'obtention d'équations fiables nécessite un nombre suffisant de mesures, réalisées à des longueurs d'onde régulièrement réparties dans le domaine de transparence du cristal. L'affinement par moindres carrés non linéaires est généralement réalisé avec la méthode du gradient ou avec l'algorithme de Levenberg-Marquardt, en ajustant tous les paramètres simultanément. Par exemple, si l'équation (5) est utilisée, l'affinement porte sur sept paramètres, $a, b, c, d, e, p$ et $q$, et ce pour chacun des indices principaux, ce qui fait quatorze paramètres pour un uniaxe et vingt et un pour un biaxe. Il est évidemment souhaitable de disposer d'un nombre de valeurs expérimentales bien supérieur au nombre des paramètres à ajuster.

L'étape d'affinement étant franchie, les directions d'accord de phase se calculent selon la procédure décrite au $\S 4.1$.

\subsubsection{Précisions et contraintes de la méthode indirecte}

Bien que le calcul des directions d'accord de phase ne pose aucune difficulté et ne nécessite aucune approximation, le résultat est souvent mauvais : il peut y avoir des écarts de plusieurs dizaines de degrés entre le calcul et l'expérience, voire un accord de phase prévu alors qu'en fait il est inexistant, et vice-versa. Le manque de fiabilité du calcul provient du manque de précision sur la mesure de l'indice de réfraction. En effet, il est nécessaire de connaitre les indices de réfraction à toute longueur d'onde de la fenêtre de transparence avec une précision de l'ordre de $10^{-4}$ pour calculer les angles d'accord de phase à $1^{\circ}$ près. Or cette précision est difficilement accessible dans la pratique pour un certain nombre de raisons :

- $\quad$ il est difficile d'usiner des prismes orientés ;

- les faces du prisme sont généralement bombées lorsque les échantillons sont millimétriques ce qui introduit des erreurs parfois importantes sur l'angle $\Delta$;

- le repérage du minimum de déviation est difficile dans l'infrarouge.

Ainsi classiquement la précision atteinte est de l'ordre de $10^{-3}$ dans le visible ; elle est encore moins bonne dans l'infrarouge. Les précisions requises pour le calcul des directions d'accord de phase nécessiteraient des prismes de 3 ou $4 \mathrm{~cm}$ de côté, ce qui n'est généralement pas compatible avec les volumes accessibles dans les premiers stades d'une cristallogenèse d'un nouveau matériau.

\subsubsection{Méthode directe}

L'échec relatif de la méthode indirecte a motivé l'émergence de techniques qui permettent une mesure directe des directions d'accord de phase.

Lorsque le nouveau matériau est obtenu sous forme de poudre, les cristallites ayant des dimensions généralement inférieures à $100 \mu \mathrm{m}$, il est impossible de mesurer les directions d'accord de phase. Néanmoins, des expériences de génération de second harmonique menées sur des poudres peuvent indiquer si l'interaction considérée est en accord de phase ou non, sans pour autant permettre la mesure des directions d'accord de phase. La méthode consiste à étudier la dépendance de l'intensité du second harmonique en fonction de certains paramètres comme l'angle entre le détecteur et le faisceau laser fondamental incident sur la poudre, l'épaisseur de la couche de poudre, la distribution de la taille des particules et le diamètre du faisceau laser fondamental [11]. En fait, cette méthode est 
surtout utilisée pour la mise en évidence du caractère non centro-symétrique d'un nouveau matériau, ce caractère étant un critère nécessaire pour avoir un tenseur $\chi^{(2)}$ dont les éléments ne sont pas tous nuls.

Pour des dimensions supérieures à quelques centaines de microns, il est possible de réaliser des mesures directes de directions d'accord de phase. Les méthodes développées jusqu'à présent sont basées sur l'utilisation d'un mono-cristal usiné en forme d'ellipsoïde [12], pour des dimensions de l'ordre de $100 \mu \mathrm{m}$, ou de sphère [13] : la sphère est la géométrie idéale, mais elle est très difficilement réalisable pour des tailles inférieures au millimètre. La méthode de la sphère est aujourd'hui une technique aboutie qui est la seule permettant la mesure directe et précise des propriétés d'accord de phase sur tout le domaine de transparence du cristal.

\subsubsection{Description de la méthode de la sphère}

La géométrie sphérique présente l'intérêt majeur de donner la possibilité d'accéder à toutes les directions du cristal en incidence normale.

Après usinage, la sphère est orientée à l'aide d'un diffractomètre à rayons $\mathrm{X}$ puis collée sur une tête goniométrique. Cette étape nécessite la connaissance du groupe de symétrie d'orientation du cristal ainsi que de ses paramètres de maille $(\boldsymbol{a}, \boldsymbol{b}, \boldsymbol{c})$. La sphère est ensuite placée au centre d'un cercle d'Euler où est focalisé le rayonnement laser. La méthode consiste à faire tourner la sphère sur ellemême grâce aux trois rotations indépendantes du cercle, $\omega_{c}, \chi_{c}$ et $\phi_{c}$, de façon à ce que le rayonnement puisse se propager successivement dans toutes les directions du cristal. Une direction d'accord de phase est détectée lorsque le rendement de conversion de l'interaction considérée est maximum. Elle est repérée par ses coordonnées d'Euler $\omega_{c}^{A P}, \chi_{c}^{A P}$ et $\phi_{c}^{A P}:$ l'orientation du repère optique $(O x, O y$, $O z$ ) étant connue par rapport au repère cristallographique $(\mathbf{a}, \mathbf{b}, \mathbf{c})$, un changement de repère permet alors de calculer les coordonnées sphériques $\left(\theta^{A P}, \phi^{A P}\right)$ de la direction d'accord de phase dans le repère optique. Ainsi, des rotations successives permettent de déterminer l'ensemble des angles d'accord de phase et les rendements de conversion associés, correspondant à l'interaction étudiée, caractérisée par les longueurs d'onde et la configuration de polarisation. Les schémas de l'expérience et du protocole correspondant sont donnés figure 11 . Le banc de mesure permet d'étudier les interactions de somme et différence de fréquences pour des faisceaux incidents dont la longueur d'onde est accordable, ce qui permet d'explorer tout le domaine de transparence du cristal. Des polariseurs et lames retard permettent de considérer différentes configurations de polarisation de façon à pouvoir étudier tous les types d'accord de phase possibles. La figure $\underline{12}$ donne l'exemple d'une mesure réalisée avec le banc de la figure $\underline{11}$ : il s'agit d'un doublage de fréquence de type II pour une longueur d'onde fondamentale de 1,596 $\mu \mathrm{m}$ dont l'accord de phase est recherché dans le plan $y$-z d'une sphère de $\mathrm{RbTiOAsO}_{4}$ (RTA) usiné en sphère. Le balayage angulaire de la figure 12 permet non seulement de déterminer l'angle d'accord de phase et le rendement de conversion associé, mais également l'acceptance angulaire interne $L \delta \theta$ de la direction d'accord de phase.

La figure 13 donne l'exemple de deux courbes d'accord de phase relatives aux plans $x-z$ et $y-z$ de $\mathrm{RbTiOPO}_{4}$ (RTP) correspondant à la génération de différence de fréquences (DFG) de type I réalisées avec différentes longueurs d'onde d'incidence [14]. Ces mesures n'ont pas été réalisées sur une sphère de RTP mais sur deux cylindres d'orientations différentes : un dont l'axe de révolution est $O y$ pour l'étude du plan $x-z$ et l'autre d'axe $O x$ pour l'étude de $y$ - $z$. Sur la figure $\underline{13}$ sont également portées les courbes calculées à partir d'indices de réfraction préalablement mesurés dans le cadre de la méthode indirecte décrite au § 4.2.1. Il apparaît des écarts importants entre le calcul et la mesure, surtout à grande longueur d'onde, ce qui corrobore les commentaires faits au sujet des limitations de la méthode indirecte. Ces courbes sont d'un intérêt pratique évident car elles donnent directement les angles d'accord de phase des interactions considérées, qui ont pu être choisies en vue d'applications particulières. C'est le cas des courbes de DFG de la figure $\underline{13}$ qui peuvent être utilisées directement pour définir les angles de taille des cristaux de RTP en vue de réaliser des oscillateurs paramétriques optiques pompés à $1,064 \mu \mathrm{m}$. 


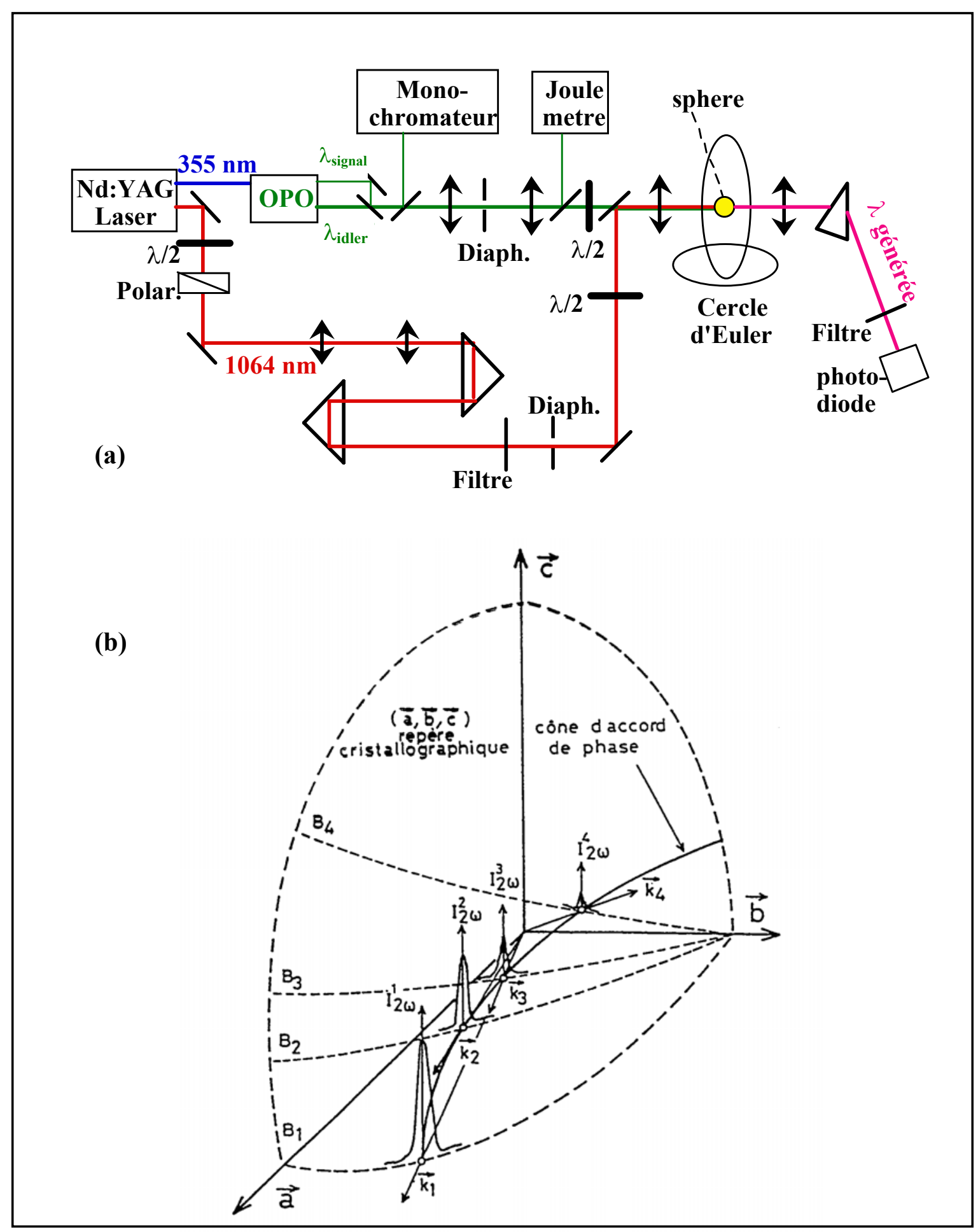

FIG. 11. Schéma de principe de la méthode de la sphère. (a) Dispositif expérimental ; la longueur d'onde générée peut être $\lambda_{g}=\frac{\lambda_{i}}{2}$ ou $\frac{\lambda_{s}}{2}$ ou $\left(\frac{1}{1064} \pm \frac{1}{\lambda_{i}}\right)^{-1}$ ou $\left(\frac{1}{1064} \pm \frac{1}{\lambda_{s}}\right)^{-1}$. (b) Description d'une nappe d'accord de phase ; les $B_{i}(i=1,2,3,4)$ sont les plans de balayage contenant les directions d'accord de phase de vecteurs d'ondes $\mathbf{k}_{i}$; les $I_{2 \omega}^{i}$ sont les intensités harmoniques correspondantes. 


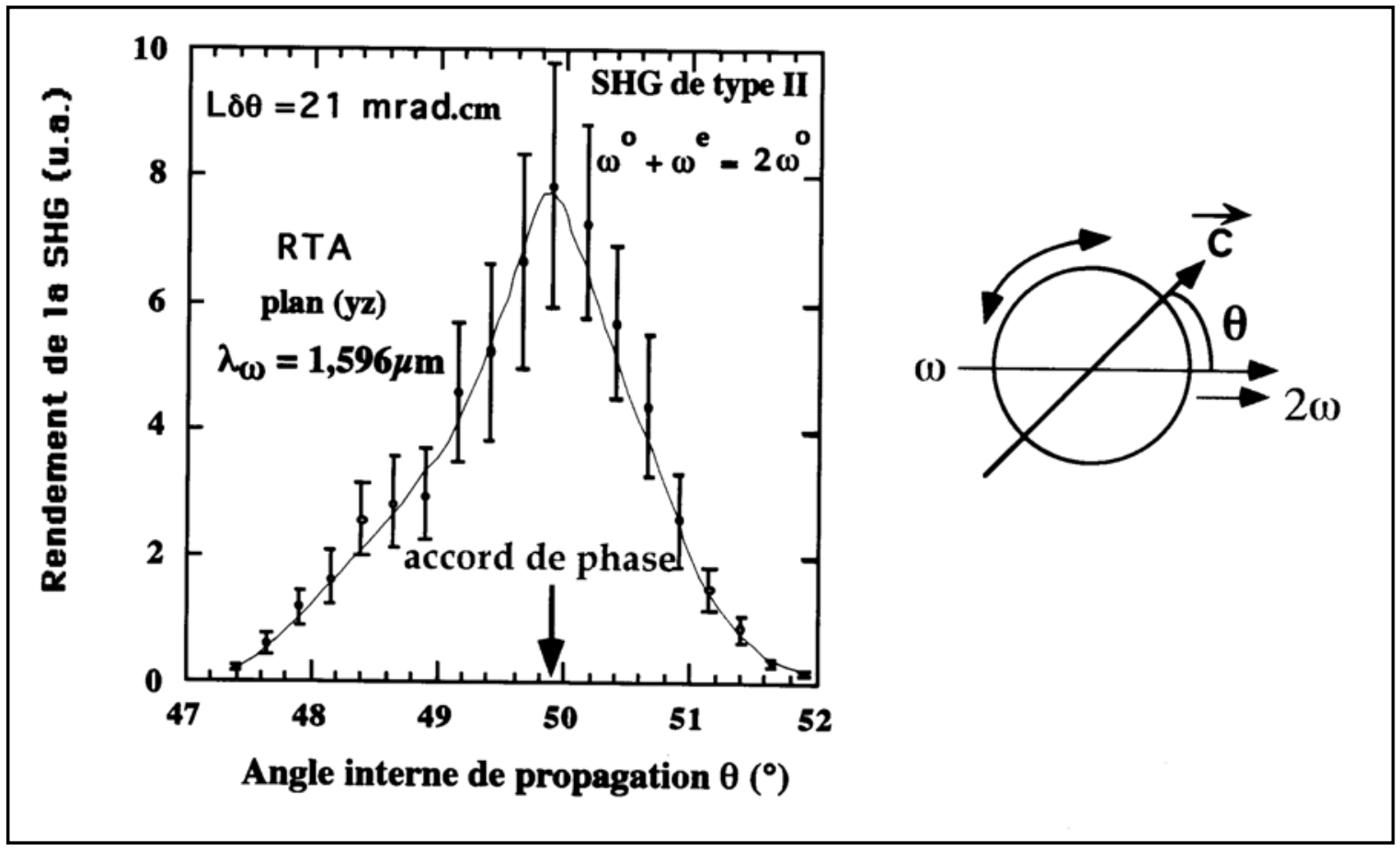

FIG. 12. Mesure du rendement de SHG en fonction de l'angle $\theta$ au voisinage de l'angle d'accord de phase. $\overrightarrow{\mathbf{c}}$ est l'axe cristallographique binaire correspondant à l'axe $O z$ du repère optique. $o$ est relatif à l'onde ordinaire, polarisée orthogonalement à $\overrightarrow{\mathbf{c}}, e$ est relatif à l'onde extraordinaire.

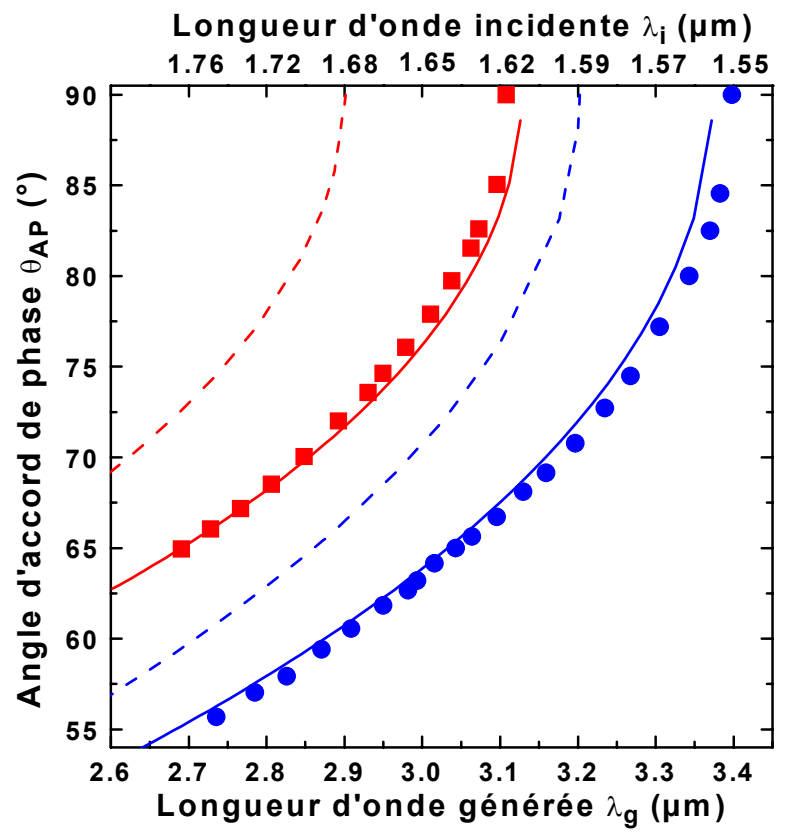

FIG. 13. Courbes d'accord de phase de RTP calculées et mesurées dans le plan $x-z(\square)$ et $y-z(\bullet)$ pour la DFG de type $\mathrm{I} \frac{1}{\lambda_{1,064 \mu \mathrm{m}}^{o}}-\frac{1}{\lambda_{i}^{o}}=\frac{1}{\lambda_{g}^{e}}$. Les courbes en pointillés sont calculées à partir d'équations de Sellmeier antérieures à ces travaux. Les courbes en traits pleins correspondent au calcul à partir des indices de réfraction déterminées grâce à un ensemble de mesures sur cylindres. 
Ces données peuvent également être exploitées afin de déterminer des équations de dispersion en longueur d'onde des indices de réfraction, ce qui permet alors de calculer les angles d'accord de phase d'interactions qui n'ont pas été considérées lors des mesures. Dans ce cas, les données peuvent être interpolées par les relations ( $(\underline{6}),(\underline{7})$ ou ( $(\underline{)})$ en utilisant des équations de dispersion, du type ()ㅜ ou (5) par exemple. Il existe toutefois une différence majeure par rapport aux mesures directes d'indice de réfraction. En effet, comme les directions d'accord de phase sont définies par des intersections de nappes des surfaces des indices, l'application d'un même facteur multiplicatif aux indices principaux ne modifie en rien les directions d'accord de phase. De ce fait, les mesures réalisées sur sphère permettent uniquement de déduire la dispersion en longueur d'onde des valeurs relatives des indices principaux, $n_{\mathrm{x}} / n_{\mathrm{y}}, n_{\mathrm{x}} / n_{\mathrm{z}}$ et $n_{\mathrm{y}} / n_{\mathrm{z}}$. Les trois indices principaux sont alors connus à une constante multiplicative près, mais avec une très bonne précision, de l'ordre de $10^{-4}$, ce qui permet de calculer les angles d'accord de phase à mieux que le degré. Par contre, si la valeur absolue d'un seul indice principal est connue à une longueur d'onde donnée, suite à une mesure sur prisme par exemple, il est alors possible de déterminer complètement les équations de dispersion : pour cela il faut prendre en compte cette donnée supplémentaire lors de l'affinement par moindres carrés. Comme dans le cas des mesures directes d'indices de réfraction décrites au § 4.2.1.1, l'affinement porte sur les paramètres des équations de dispersion, les équations déduites étant d'autant plus fiables pour le calcul des angles d'accord de phase que les interactions choisies pour les mesures sur sphère solliciteront tous les indices principaux à des longueurs d'onde bien réparties sur tout le domaine de transparence du cristal à l'étude. À titre d'exemple, la figure 13 montre les courbes d'accord de phase calculées à partir des indices de réfraction modélisés par l'équation (므) et obtenus par interpolation de 268 mesures d'angle d'accord de phase.

\subsubsection{Bilan sur les performances de la méthode directe}

L'incertitude sur l'angle d'accord de phase mesuré dépend de paramètres tels que la précision des angles des trois rotations d'Euler $\left(\leq 0,1^{\circ}\right)$, l'excentricité du cercle d'Euler $(\leq 0,1 \mathrm{~mm})$, la précision d'orientation de la sphère $\left(\leq 0,1^{\circ}\right)$ ou le centrage de la sphère par rapport au rayonnement incident. L'asphéricité $(\Delta d / d \leq 1 \%$, où $d$ est le diamètre) contribue également à l'incertitude. D'autre part, lorsque que le diamètre de la sphère est petit, ce qui est souvent le cas, l'acceptance angulaire est large de sorte que l'estimation du maximum de rendement de conversion est difficile. L'incertitude résultante typique est alors de l'ordre de $\pm 1^{\circ}$. Pour augmenter la précision des mesures, il est astucieux de mettre à profit la symétrie des cônes d'accord de phase, ce qui en pratique n'est réalisable que lorsqu'un accord de phase est permis dans un plan principal de la surface des indices, $x-y, x-z$ ou $y-z$, et que le coefficient effectif associé n'est pas nul : dans ce cas, il est possible de mesurer l'angle entre les deux directions symétriques, dont la moitié de la valeur donne directement l'angle d'accord de phase avec une excellente précision, de l'ordre de $\pm 0,5^{\circ}$.

L'usinage et le polissage de la sphère, son orientation ainsi que toute la procédure d'alignement et de centrage des optiques et de la sphère constituent des étapes délicates. D'autre part, il est nécessaire de réaliser au moins deux collages différents, orientés selon deux des trois axes principaux du repère optique, afin de prospecter tout le volume de la sphère : en effet pour un collage donné, il existe un angle solide inaccessible au rayonnement du fait de la surface de collage et de l'écrantage par certains éléments mécaniques. Malgré ces difficultés, la méthode de la sphère est extrêmement performante car elle permet de mesurer directement toute direction d'accord de phase ainsi que le rendement de conversion et les tolérances angulaires associés, avec un seul échantillon du cristal à l'étude et avec comme seule donnée initiale sa classe de symétrie d'orientation. Un autre intérêt de la méthode est la détermination d'équations de dispersion très fiables des indices de réfraction. 


\section{5. ÉTUDE DU TENSEUR DE SUSCEPTIBILITÉ ÉLECTRIQUE DE DEUXIÈME ORDRE $\chi^{(2)}$}

Le tenseur de susceptibilité électrique de second ordre $\chi^{(2)}$ est un tenseur polaire de rang 3 qui possède vingt-sept éléments indépendants dans le cas général. Ces éléments sont notés $\chi_{i j k}$ où $(i, j, k)$ sont les indices cartésiens relatifs au repère optique $(x, y, z)$. Le tenseur $\chi^{(2)}$ se représente par une matrice $3 \times 9$ comme le montre la figure 14 . Pour la SHG, le tenseur est symétrique vis-à-vis des deux derniers indices cartésiens, soit: $\chi_{i j k}(2 \omega=\omega+\omega)=\chi_{i k j}(2 \omega=\omega+\omega)$; ainsi le nombre d'éléments indépendants se réduit à dix-huit dans le cas général. Le tenseur $\chi^{(2)}$ est alors souvent écrit en notation contractée avec la convention classique : $x x \equiv 1, y y \equiv 2, z z \equiv 3, y z=z y \equiv 4, x z=z x \equiv 5, x y=y x \equiv 6$. Si les pulsations $\omega$ et $2 \omega$ sont situées loin des résonances, de sorte qu'il est possible de considérer que le tenseur $\chi^{(2)}$ n'est pas dispersif (approximation de Kleinmann), alors $\chi^{(2)}$ devient symétrique vis-à-vis des trois indices cartésiens et ne possède donc plus que dix éléments indépendants. Ce résultat est généralisable à toute interaction à trois ondes, somme ou différence de fréquences. Le nombre d'éléments indépendants dépend également de la symétrie d'orientation du milieu en vertu du principe de Neumann [2]. Par exemple, tous les éléments sont nuls pour toute classe cristalline centrosymétrique ; pour la classe $m m 2$, qui est celle de nombreux cristaux non linéaires, il y a sept éléments indépendants : $\chi_{\mathrm{xxz}}, \chi_{\mathrm{xzx}}, \chi_{\mathrm{yyz}}, \chi_{\mathrm{yzy}}, \chi_{\mathrm{zxx}}, \chi_{\mathrm{zyy}}$ et $\chi_{\mathrm{zzz}}$. Ce nombre est réduit à cinq dans le cas de la SHG et à trois lorsque l'approximation de Kleinmann est utilisée.

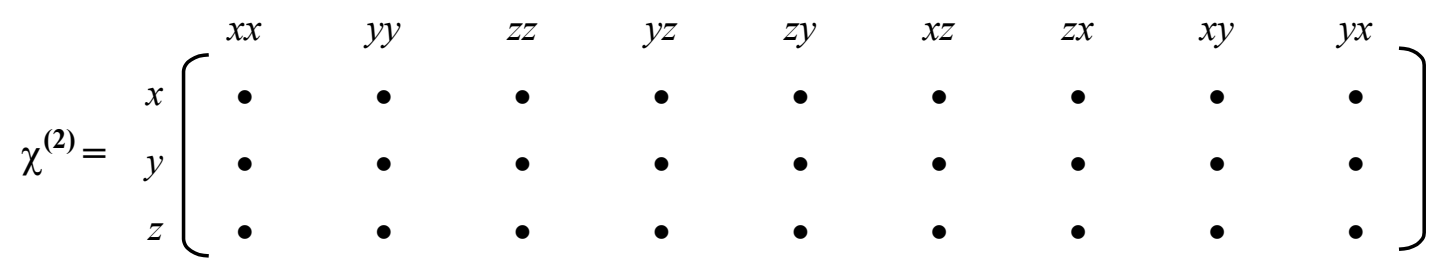

Fig. 14. Cas général de la représentation matricielle du tenseur $\chi^{(2)}$ dans le repère optique $(x, y, z)$.

Pour un même cristal, il existe souvent une grande disparité entre les valeurs de coefficients non linéaires publiées par différents auteurs. Les mesures n'étant pas réalisées avec les mêmes échantillons, la différence de résultat peut provenir d'une fluctuation de la qualité cristalline, mais c'est souvent le type de méthode qui est en cause. De plus, il existe plusieurs conventions d'écriture de la polarisation non linéaire en fonction de la susceptibilité électrique, ce qui vient compliquer la comparaison : par exemple certains auteurs travaillent avec des coefficients non linéaires de second ordre définis par $d_{i j k}=\chi_{i j k} / 2$.

\subsection{Coefficient effectif, tenseur de susceptibilité électrique et tenseur champ}

La susceptibilité électrique de second ordre intervient dans le coefficient effectif, $\chi_{\text {eff }}$, qui a été défini au § 3.1. Dans le cadre de la mesure des éléments indépendants du tenseur $\chi^{(2)}$, il est intéressant d'utiliser le formalisme du tenseur champ, qui permet de faire apparaitre la contribution de chacun des $\chi_{i j k}$ dans l'expression de $\chi_{\text {eff }}$, soit pour la somme de fréquence $\omega_{3}=\omega_{1}+\omega_{2}[\underline{15}]$ :

$$
\chi_{\text {eff }}\left(\omega_{3}=\omega_{1}+\omega_{2}, \theta, \phi\right)=\sum_{i j k} F_{i j k}\left(\omega_{3}=\omega_{1}+\omega_{2}, \theta, \phi\right) \cdot \chi_{i j k}\left(\omega_{3}=\omega_{1}+\omega_{2}\right)
$$

avec

$$
F_{i j k}\left(\omega_{3}=\omega_{1}+\omega_{2}, \theta, \phi\right)=e_{i}\left(\omega_{3}, \theta, \phi\right) e_{j}\left(\omega_{1}, \theta, \phi\right) e_{k}\left(\omega_{2}, \theta, \phi\right)
$$

$F_{i j k}\left(\omega_{3}=\omega_{1}+\omega_{2}, \theta, \phi\right)$ représente le degré de sollicitation de l'élément $\chi_{i j k}\left(\omega_{3}=\omega_{1}+\omega_{2}\right)$ dans la direction considérée $(\theta, \phi)$ et pour une configuration de polarisation donnée $\left(\mathbf{e}_{1}, \mathbf{e}_{2}, \mathbf{e}_{3}\right)$. 
La polarisation non linéaire s'écrit donc comme le produit contracté de deux tenseurs polaires de rang 3: le tenseur $\chi^{(2)}$, caractéristique de la non linéarité quadratique du cristal, et le tenseur $F^{(2)}$ qui dépend uniquement de la configuration de polarisation des ondes. La symétrie de $F^{(2)}$ est donc imposée par le tenseur des indices de réfraction et le choix des modes propres de polarisation comme le montre la figure $\underline{15}$ dans le cas des cristaux uniaxes et des plans principaux des cristaux biaxes. Notons qu'il n'existe aucune relation entre les éléments du tenseur champ pour une direction de propagation contenue hors des plans principaux d'un cristal biaxe du fait de l'absence d'orthogonalité entre les modes propres de polarisation; le tenseur champ possède alors vingt-sept éléments indépendants.

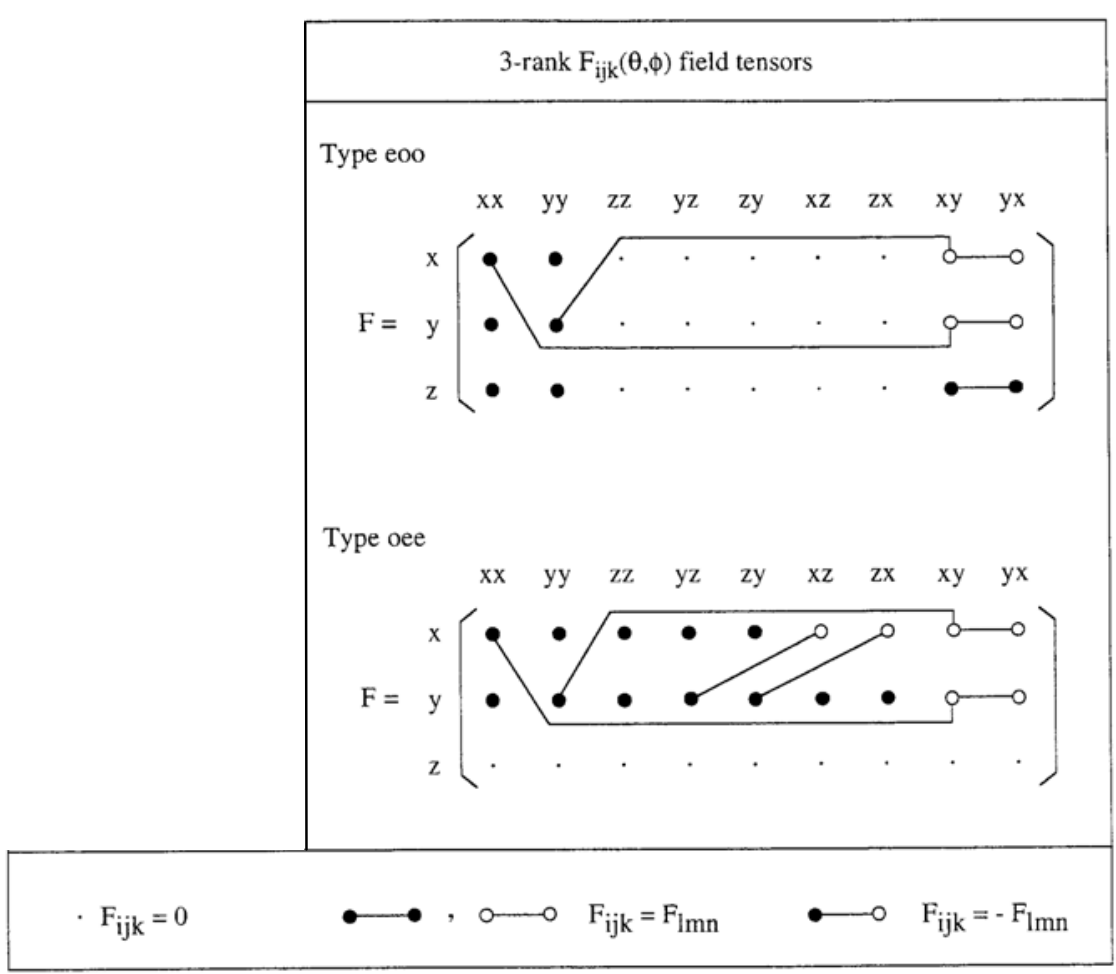

FIG. 15. Représentation matricielle du tenseur champ dans le repère optique $(x, y, z)$ pour les interactions couplant une onde extraordinaire à deux ondes ordinaires (type eoo) et les interactions couplant deux ondes extraordinaires à une onde ordinaire (type oee). Les angles $(\theta, \phi)$ sont les coordonnées sphériques d'une direction de propagation quelconque dans les cristaux uniaxes ou dans les plans principaux $x-z$ et $y-z$ des cristaux biaxes. Il faut intervertir $o$ et $e$ pour le plan $x-y$ des cristaux biaxes.

\subsection{Mesure des coefficients du tenseur $\chi^{(2)}$}

Très généralement, les coefficients non linéaires sont mesurés en situation de SHG hors accord de phase ou en accord de phase, ce qui définit deux types de stratégie. Toutes les méthodes reposent sur la mesure du rendement de conversion à partir duquel est déterminé le carré du coefficient effectif, $\chi_{\text {eff }}^{2}$. Ainsi, seule la valeur absolue, $\left|\chi_{\text {eff }}\right|$, est accessible. Par le biais de plusieurs mesures réalisées dans différentes directions de propagation il est possible de déterminer la valeur absolue et le signe relatif des coefficients indépendants du tenseur.

Il s'agit de se placer dans des situations où le rendement de conversion de l'interaction non linéaire se calcule le plus aisément possible, en travaillant par exemple avec des ondes planes et en situation de pompe non dépeuplée. Ces calculs nécessitent de connaître au préalable les indices de réfraction, une précision de l'ordre de $10^{-3}$ étant généralement suffisante. Quelle que soit la méthode choisie, la mesure en absolu des coefficients non linéaires nécessite la connaissance la plus précise 
possible du faisceau à $\omega$ en terme d'énergie, de profil spatial, transverse et longitudinal, et de profil temporel. Cette étape primordiale représente une part importante et souvent ingrate du travail de mesure. Cet aspect est beaucoup moins crucial pour une mesure en relatif : dans ce cas, le rendement de conversion d'un cristal étalon, dont le coefficient effectif est connu, est mesuré dans les mêmes conditions expérimentales.

\subsubsection{Mesure par SHG hors accord de phase}

Il existe deux méthodes procédant en hors accord de phase : la technique du prisme translaté et la méthode des franges de Maker.

\subsubsection{Méthode du prisme translaté}

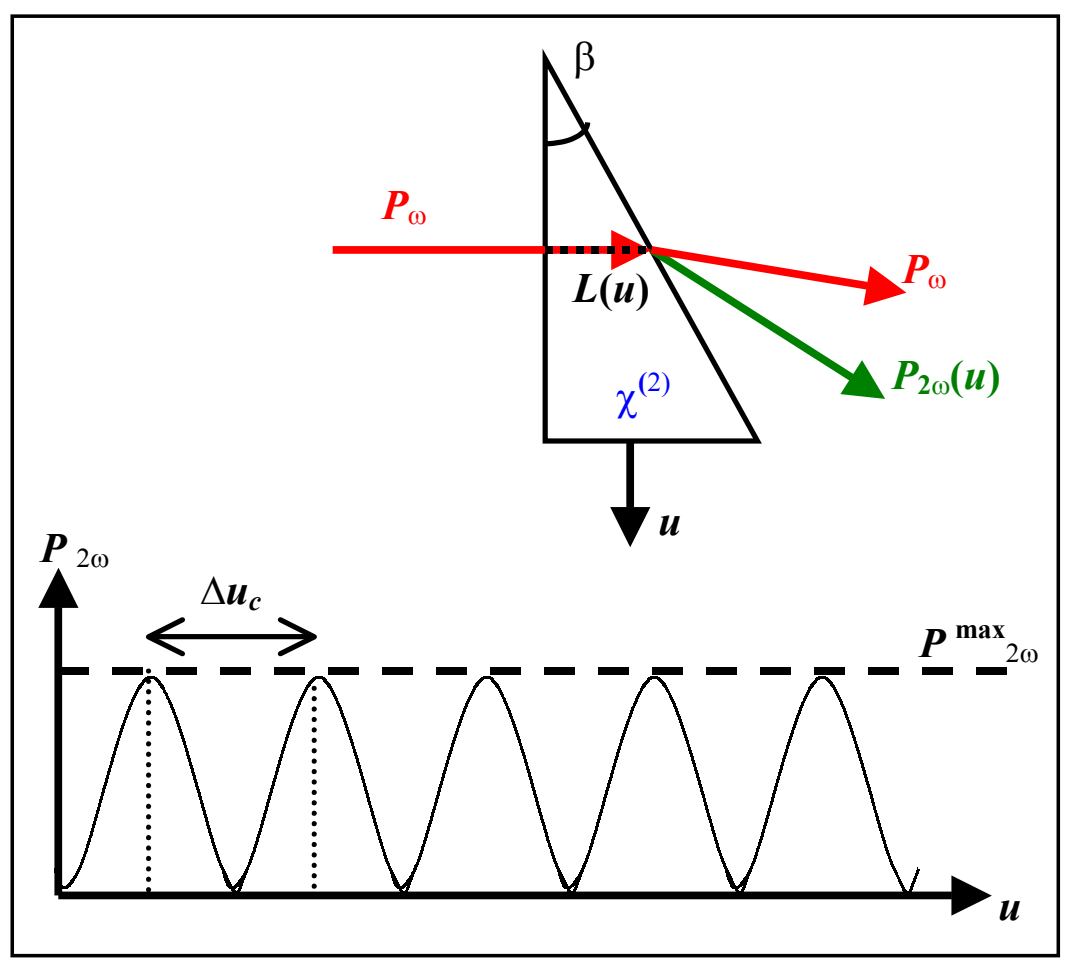

FIG. 16. Schéma de principe de la méthode du prisme translaté. $P_{\omega}$ et $P_{2 \omega}$ sont les puissances fondamentale et harmonique. $\beta$ est l'angle du prisme. $L(u)$ est la distance d'interaction qui dépend de $u . \Delta u_{c}$ est la distance entre deux franges.

La méthode consiste à mesurer le rendement de conversion en fonction de l'épaisseur traversée par le rayonnement. La variation d'épaisseur, $L(u)$, est obtenue en translatant l'échantillon, usiné en forme de prisme d'angle $\beta$, selon une direction u orthogonale à l'axe du faisceau incident [16], comme le montre la figure 16 . À une configuration de polarisation donnée, le paramètre $\Delta k$ de l'interaction reste fixe quel que soit $u$, de sorte que la puissance générée varie sinusoïdalement en fonction de la seule variable $u$. La mesure de la période, $\Delta u_{c}$, et de la puissance maximale générée, $P_{2 \omega}^{\max }$, permet alors de calculer aisément la valeur absolue du coefficient effectif, soit compte tenu de l'expression (2) établie au $§ 3.2$ :

$$
\left|\chi_{\text {eff }}\right|=\sqrt{\frac{P_{2 \omega}^{\max }}{\frac{4 A}{\pi^{2}} \frac{\left(P_{t o t}^{\omega}\right)^{2}}{w_{o}{ }^{2}} L_{c}{ }^{2}}}
$$


avec

$$
A=\frac{9462}{\lambda_{\omega}^{2}} \frac{2 N-1}{N} \frac{T_{3}^{2 \omega} T_{1}^{\omega} T_{2}^{\omega}}{n_{3}^{2 \omega} n_{1}^{\omega} n_{2}^{\omega}}
$$

et $L_{\mathrm{c}}$, la longueur de cohérence de l'interaction reliée à l'angle du prisme $\beta$ par :

$$
L_{c}=\frac{\Delta u_{c} \tan \beta}{2}
$$

L'angle $\beta$ doit être choisi en fonction du rayon $w_{0}$ du faisceau de mesure. Il est en effet nécessaire que la différence entre les trajets parcourus par les différents rayons soit très inférieure à la longueur de cohérence, soit : $\tan \beta \ll \frac{L_{c}}{2 w_{o}}$. Ainsi pour $L_{\mathrm{c}}=10 \mu \mathrm{m}$ et $w_{o}=20 \mu \mathrm{m}$, il faut $\beta \ll 14^{\circ}$, typiquement $1^{\circ}$.

De plus il existe une double contrainte sur $w_{o}$, vis-à-vis d'une part de la densité de puissance et d'autre part du caractère onde plane. En effet, l'interaction étant réalisée hors accord de phase, il est souvent nécessaire, pour pouvoir détecter le rayonnement à $2 \omega$, de focaliser le faisceau à $\omega$ de façon à travailler avec une densité de puissance de l'ordre de $100 \mathrm{MW} / \mathrm{cm}^{2}$. La focalisation est alors réalisée au centre du prisme, mais il faut que l'épaisseur traversée soit inférieure à deux fois la longueur de Rayleigh de sorte que les ondes réfractées dans le prisme soient parallèles. L'épaisseur $e$ du prisme selon l'axe du faisceau doit donc vérifier : $e \ll \frac{2 \pi w_{o}{ }^{2} n}{\lambda_{\omega}}$, où $n$ est l'indice de réfraction du cristal; pour $w_{\mathrm{o}}=20 \mu \mathrm{m}, n=2$ et $\lambda_{\omega}=1 \mu \mathrm{m}$, il faut $e \ll 5 \mathrm{~mm}$, typiquement $1 \mathrm{~mm}$.

Pour ces valeurs typiques de $\beta, w_{o}$ et $e$, il y a des réflexions multiples dont les interférences viennent brouiller le réseau de franges obtenues par translation. En effet il y a recouvrement entre les faisceaux aller et retour si $e \leq \frac{2 w_{o}}{\tan 2 \beta}$, soit pour des épaisseurs inférieures à $2 \mathrm{~mm}$ pour $w_{o}=20 \mu \mathrm{m}$ et $\beta=0,5^{\circ}$. Dans ce cas il peut s'avérer utile de traiter anti-reflet les faces du prisme.

A priori, il suffit de trois prismes orientés selon les trois directions du repère optique pour être en mesure de déterminer tous les éléments indépendants du tenseur $\chi^{(2)}$, le choix de la polarisation du rayonnement incident à $\omega$ et de la polarisation d'analyse à $2 \omega$ étant totalement libre. Ainsi, une propagation selon l'axe $O x, O y$ ou $O z$, permet respectivement la détermination des coefficients impliquant les indices cartésiens $y$ et $z, x$ et $z$ ou $x$ et $y$.

La détermination de $L_{\mathrm{c}}$ est utile en soit pour la fabrication des cristaux pour le quasi-accord de phase, l'inversion du signe du coefficient effectif devant être réalisée tous les $L_{c}$. La méthode du prisme translaté est donc la technique de mesure la plus directe dans ce cas.

\subsubsection{Méthode des franges de Maker}

La méthode des franges de Maker consiste à mettre en rotation l'échantillon parallélépipédique par rapport à l'axe du faisceau à $\omega$ comme le montre la figure 17 . La mesure consiste alors à enregistrer la puissance générée à $2 \omega$ en fonction de l'angle de rotation $\alpha$. En incidence oblique et lorsqu'il n'y a pas accord de phase, les vecteurs d'onde réfractés à $\omega$ et $2 \omega$ ne sont pas colinéaires, et leurs directions dépendent de l'angle d'incidence [6]. Il s'en suit une variation du désaccord de phase qui se traduit par l'oscillation de la puissance harmonique avec l'angle d'incidence [17]. Une expérience typique de franges de Maker nécessite un balayage angulaire de quelques dizaines de degrés de part et d'autre de la normale à la face d'entrée, de sorte que l'équation (2) ne peut plus être utilisée. Les expressions adaptées, correspondant aux différents plans principaux des cristaux uniaxes et biaxes, peuvent être établies à partir des équations de la référence [16]. Par exemple, dans le cas simple d'une SHG 
couplant trois ondes extraordinaires dans le plan principal d'un cristal uniaxe, la puissance harmonique en fonction de l'angle $\alpha$ est donnée par :

$$
\begin{gathered}
P^{2 \omega}(\alpha)=C(\alpha) \cos \alpha\left(P_{t o t}^{\omega}\right)^{2} \frac{L^{2}}{w_{o}{ }^{2}} \operatorname{sinc}^{2}(\psi(\alpha)) \\
C(\alpha)=\frac{2366}{\lambda_{\omega}{ }^{2}} \frac{2 N-1}{N} \frac{T_{2 \omega}(\alpha) T_{\omega}{ }^{2}(\alpha)}{a_{2 \omega}(\alpha) a_{\omega}{ }^{2}(\alpha)} \chi_{33}{ }^{2} \\
a_{i}(\alpha)=\sqrt{{n_{i}}^{2}-\sin ^{2} \alpha} \quad \text { avec } i \equiv \omega, 2 \omega \\
\psi(\alpha)=\frac{2 \pi L}{\lambda}\left[a_{2 \omega}(\alpha)-a_{\omega}(\alpha)\right]
\end{gathered}
$$

Les équations précédentes permettent d'exploiter un enregistrement expérimental de franges de Maker en vue de la détermination de $\left|\chi_{33}\right|$ : le cristal uniaxe à l'étude est usiné en parallélépipède dont les normales aux faces sont les axes du repère optique; les faisceaux se propagent orthogonalement à l'axe $O z$ qui est également l'axe de rotation du cristal et la direction de polarisation des faisceaux à $\omega$ et $2 \omega$. Les données expérimentales sont interpolées avec $\chi_{33}{ }^{2}$ comme paramètre ajustable à déterminer. L'oscillation dépendant fortement des indices de réfraction, il est souvent nécessaire d'en ajuster leurs valeurs pour que l'interpolation soit correcte.

Comme la méthode du prisme translaté, la méthode des franges de Maker permet de mesurer chacun des coefficients indépendants $\chi_{i j k}$ du fait du libre choix des directions de propagation et de polarisation.

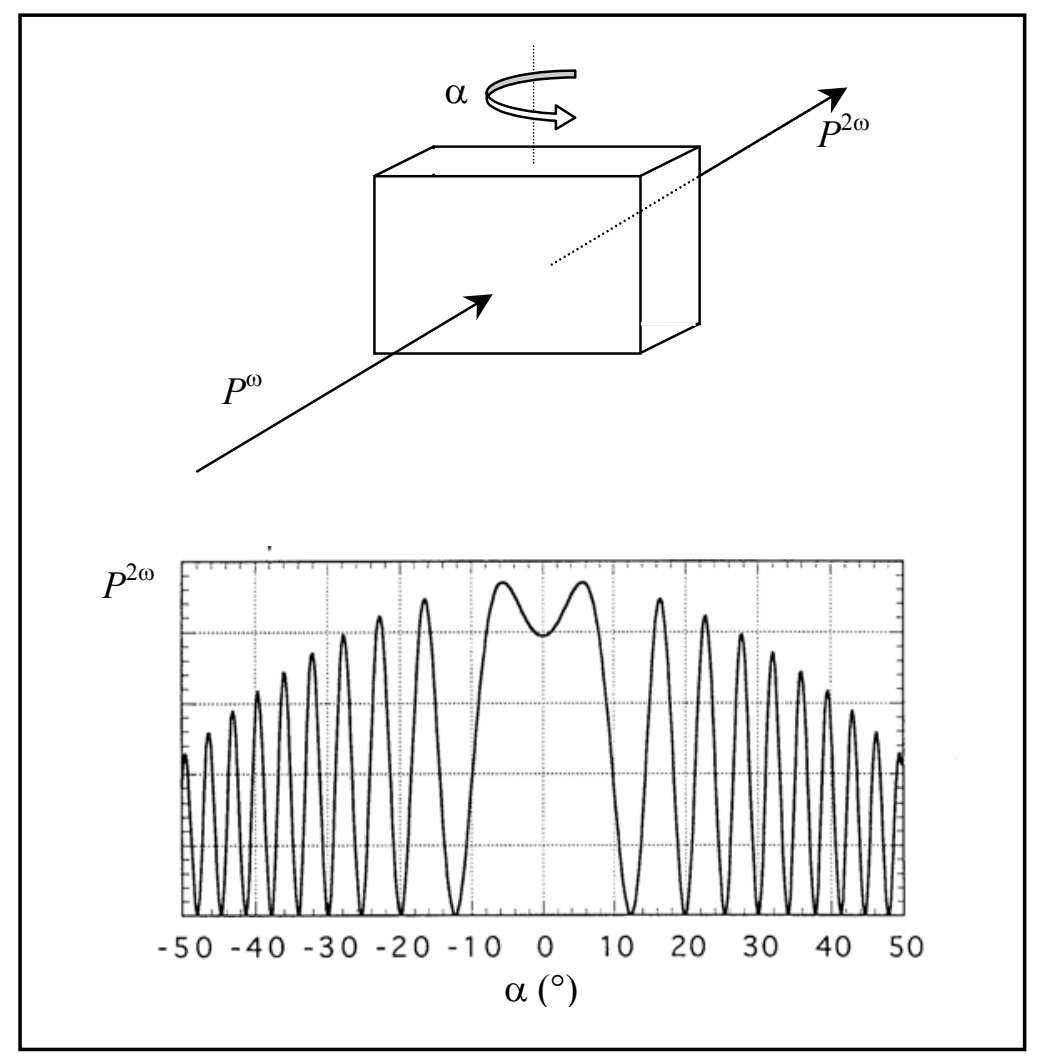

FIG 17. Schéma de principe de la méthode des franges de Maker. $\alpha$ est l'angle entre le faisceau incident de puissance $P_{\omega}$ et la normale à la face d'entrée. $P_{2 \omega}$ est la puissance harmonique générée. 


\subsubsection{Bilan des méthodes par SHG hors accord de phase}

Le grand intérêt des deux méthodes basées sur une interaction hors accord de phase est qu'il est possible de mesurer de façon indépendante la valeur absolue de chaque élément indépendant du tenseur. Par contre il est difficile de déterminer le signe relatif de ces éléments. Pour cela il faut considérer des plans de balayage et des configurations de polarisation tels que le coefficient effectif est une fonction angulaire de plusieurs coefficients. Le système de franges est alors complexe et difficilement exploitable.

La faiblesse du rendement de conversion est l'inconvénient majeur de ces méthodes. Pour remédier à ceci, il est souvent nécessaire de travailler en régime impulsionnel ce qui permet d'atteindre des densités de puissance importantes. La contrepartie est qu'il faut alors connaître parfaitement le profil temporel de l'impulsion. Cette difficulté est contournée dans la pratique en réalisant les mesures en relatif par rapport à un cristal étalon. La précision couramment atteinte est alors de l'ordre de $10 \%$ pour la valeur relative des coefficients de $\chi^{(2)}$ par rapport à ceux du cristal étalon.

\subsubsection{Mesure par SHG en accord de phase}

Comme dans le cas des expériences de SHG hors accord de phase, la détermination des coefficients non linéaires passe par des déterminations de coefficient effectif à partir de mesures de rendement de conversion dans différentes directions d'accord de phase. Les expériences sont réalisées avec une série de lames parallélépipédiques d'orientations différentes ou avec un seul échantillon usiné en forme de sphère.

Le choix des types d'accord de phase et des directions de propagation devant conduire à la détermination du signe relatif et de la valeur absolue des coefficients non linéaires indépendants nécessite une analyse préalable du coefficient effectif, ce qui est facilité par le formalisme du tenseur champ.

Nous prenons comme exemple le cas des cristaux appartenant à la classe de symétrie d'orientation $\mathrm{mm} 2$. C'est la classe cristalline d'un grand nombre de cristaux à propriétés optiques non linéaires comme : $\mathrm{KTiOPO}_{4}$ et ses isotypes, $\mathrm{KNbO}_{3}, \mathrm{LiB}_{3} \mathrm{O}_{5}, \mathrm{LiInS}_{2}$ et ses isotypes, 2A5NPDP et ses isotypes. Le tenseur $\chi^{(2)}$ correspondant a cinq coefficients indépendants dans le cas de la SHG : $\chi_{15}$, $\chi_{24}, \chi_{31}, \chi_{32}$ et $\chi_{33}$. Le coefficient effectif est alors donné par :

$$
\chi_{\text {eff }}=F_{15} \chi_{15}+F_{24} \chi_{24}+F_{31} \chi_{31}+F_{32} \chi_{32}+F_{33} \chi_{33}
$$

Les paramètres $\chi_{\text {eff }}$ et $F_{i j}$ dépendent de $(\theta, \Phi, \omega, 2 \omega)$ et les $\chi_{i j}$ sont à la pulsation $2 \omega$. Les cinq coefficients $F_{i j}$ du tenseur champ de KTP calculés à partir des indices de réfraction à $1,32 \mu \mathrm{m}$ et $0,66 \mu \mathrm{m}$ sont donnés en fonction de la coordonnée sphérique $\phi$ de la direction d'accord de phase des SHG 1,32 $\mu \mathrm{m} \rightarrow 0,66 \mu \mathrm{m}$ de type II (Fig. 18) et de type I (Fig. 19).

Il apparait sur la figure $\underline{18}$ que $\left|\chi_{15}\right|$ et $\left|\chi_{24}\right|$ peuvent être déterminés de façon indépendante dans les directions d'accord de phase situées respectivement dans le plan $\phi=90^{\circ}$, c'est-à-dire le plan $y-z$, et le plan $\phi=0^{\circ}$, soit $x-z$. Ayant déterminé la valeur absolue des coefficients effectifs correspondants, $\left|\chi_{\text {eff }}^{I I}\left(\phi=90^{\circ}\right)\right|$ et $\chi_{\text {eff }}^{I I}\left(\phi=0^{\circ}\right)$, par mesure de rendement de conversion, il vient :

$$
\begin{aligned}
& \left|\chi_{15}(2 \omega)\right|=\left|\frac{\chi_{e f f}^{I I}\left(\omega, 2 \omega, \phi=90^{\circ}\right)}{F_{15}^{I I}\left(\omega, 2 \omega, \phi=90^{\circ}\right)}\right| \\
& \left|\chi_{24}(2 \omega)\right|=\left|\frac{\chi_{e f f}^{I I}\left(\omega, 2 \omega, \phi=0^{\circ}\right)}{F_{24}^{I I}\left(\omega, 2 \omega, \phi=0^{\circ}\right)}\right|
\end{aligned}
$$




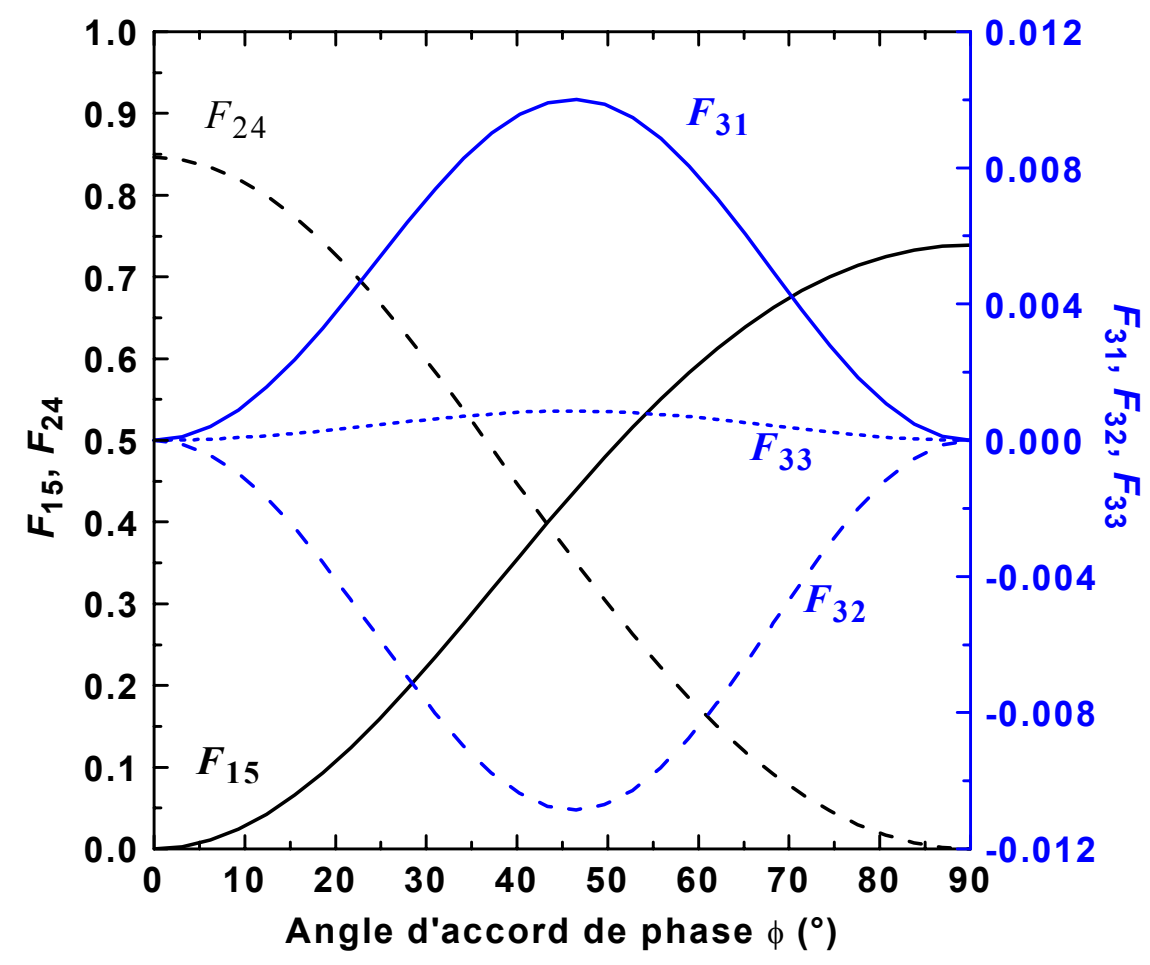

FIG. 18. Coefficients du tenseur champ relatif à la SHG de type II $1,32 \mu \mathrm{m} \rightarrow 0,66 \mu \mathrm{m}$ dans KTP, calculés en fonction de la coordonnée sphérique $\phi$ des directions d'accord de phase.

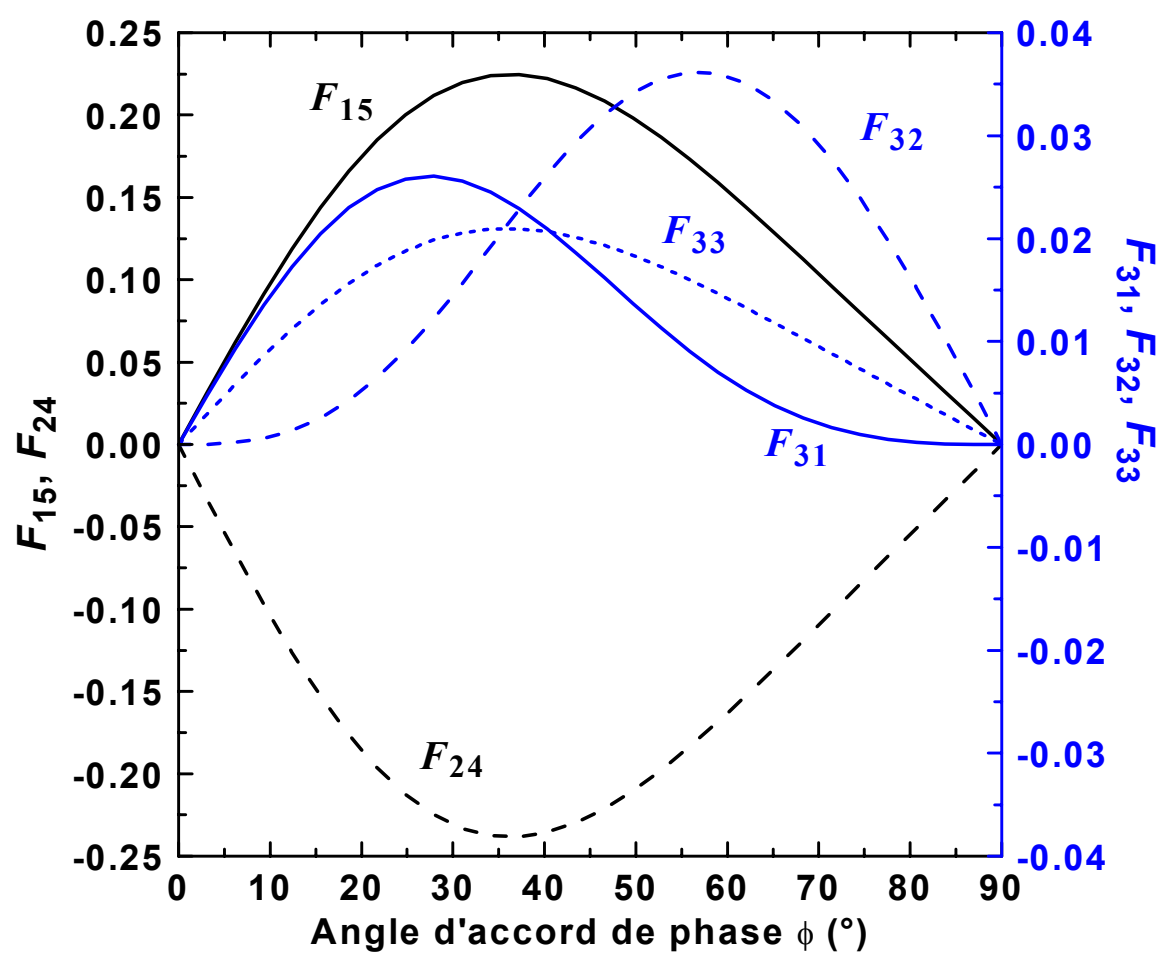

FIG. 19. Coefficients du tenseur champ relatif à la SHG de type I $1,32 \mu \mathrm{m} \rightarrow 0,66 \mu \mathrm{m}$ dans KTP, calculés en fonction de la coordonnée sphérique $\phi$ des directions d'accord de phase. 
La figure 18 montre également qu'il est possible de déterminer le signe relatif de $\chi_{15}$ et $\chi_{24}$ en réalisant des mesures de rendement de conversion de la SHG de type II à différentes directions d'accord de phase. En effet, les coefficients $F_{31}, F_{32}$ et $F_{33}$ à leur maximum étant inférieurs de deux ordres de grandeur par rapport à $F_{15}$ et $F_{24}$, le coefficient effectif dépend majoritairement de $\chi_{15}$ et $\chi_{24}$. Les coefficients $F_{15}$ et $F_{24}$ étant de même signe, le coefficient effectif sera maximum ou minimum à la direction d'accord de phase pour laquelle $F_{15}=F_{24}$ suivant que $\chi_{15}$ et $\chi_{24}$ auront respectivement des signes identiques ou opposés. Cette démarche a été suivie pour montrer que $\chi_{15}$ et $\chi_{24}$ sont de même signe dans le cas de KTP comme le montre clairement les courbes de la figure 20 [18]. Les mesures ont été réalisées à l'aide d'une sphère de KTP dans le cas de la SHG $1,064 \mu \mathrm{m} \rightarrow 0,532 \mu \mathrm{m}$, pour laquelle l'évolution des $F_{i j}$ est très semblable à celle de la figure $\underline{18}$. Ce genre de mesure aurait été très dissuasive sans sphère, car il aurait été nécessaire d'usiner plusieurs parallélépipèdes dans des directions d'accord de phase différentes, sachant que ces directions sont très mal calculées lorsque les équations de dispersion des indices de réfraction n'ont pas été fiabilisées.

Les figures $\underline{18}$ et 19 montrent qu'il est très difficile de déterminer $\chi_{31}, \chi_{32}$ et $\chi_{33}$ à partir de SHG en accord de phase. Néanmoins, il est possible de déterminer la valeur absolue et le signe relatif de $\chi_{33}$ connaissant la valeur absolue et le signe relatif de $\chi_{15}$ et $\chi_{24}$ et en se plaçant dans l'approximation de Kleinmann, qui stipule : $\chi_{31}=\chi_{15}$ et $\chi_{32}=\chi_{24}$. La méthode consiste dans un premier temps à mesurer le rendement de conversion de la SHG de type I à son maximum d'efficacité, $\eta_{\max }$. Cette mesure permet de déterminer la valeur absolue du coefficient effectif $\left|\chi_{\text {eff }}^{I}\left(\phi_{\eta_{\max }}\right)\right|$, à partir de laquelle $\chi_{33}$ peut être calculé, soit :

$$
\chi_{33}=\frac{\chi_{\text {eff }}^{I}-\chi_{15}\left(F_{15}^{I}+F_{31}^{I}\right)-\chi_{24}\left(F_{24}^{I}+F_{32}^{I}\right)}{F_{33}^{I}}
$$

Il y a donc deux valeurs possibles pour $\chi_{33}$ suivant le signe relatif de $\chi_{\text {eff }}^{I}$ et de $\left(\chi_{15}, \chi_{24}\right)$. Pour la commodité de l'exposé, choisissons arbitrairement $\chi_{15}$ et $\chi_{24}$ positifs. L'expérience conduit alors aux deux valeurs suivantes pour $\chi_{33}: 39,5 \pm 2 \mathrm{pm} / \mathrm{V}$ pour $\chi_{\text {eff }}^{I}>0$ et $10,7 \pm 0,5 \mathrm{pm} / \mathrm{V}$ pour $\chi_{\text {eff }}^{I}<0$ [1] $]$. Dans les deux hypothèses le signe de $\chi_{33}$ est positif, c'est-à-dire identique à celui de $\chi_{15}$ et $\chi_{24}$ : les signes relatifs des coefficients indépendants du tenseur $\chi^{(2)}$ sont donc établis. Le choix entre les deux valeurs possibles de $\chi_{33}$ peut être fait à partir de la mesure du rendement de SHG de type I en fonction des directions d'accord de phase. Comme précédemment, ce type d'expérience est largement facilité par l'utilisation d'une sphère. Les points expérimentaux sont tracés figure 21 . L'accord entre calcul et expérience correspond nettement au cas où $\left|\chi_{33}\right|=10,7 \pm 0,5 \mathrm{pm} / \mathrm{V}$.

Il est possible d'augmenter le nombre de coefficients déterminés de façon indépendante en complétant les mesures de SHG par des expériences de différence de fréquences (DFG). Par exemple, les coefficients $F_{31}, F_{32}, F_{33}, F_{15}$ et $F_{24}$ de la DFG de type I, dont la configuration de polarisation est $\left(\mathbf{e}_{\omega_{1}}^{+}, \mathbf{e}_{\omega_{2}}^{-}, \mathbf{e}_{\omega_{3}}^{-}\right)$, ont les mêmes allures respectivement que les coefficients $F_{15}, F_{24}, F_{33}, F_{31}$ et $F_{32}$ de la SHG de type II $\left(\mathbf{e}_{2 \omega}^{-}, \mathbf{e}_{\omega}^{+}, \mathbf{e}_{\omega}^{-}\right)$donnés figure $\underline{18}$ [15]. Ainsi, $\chi_{31}$ et $\chi_{32}$ peuvent être déterminés en valeur absolue et en signe relatif avec la même stratégie que celle décrite pour $\chi_{15}$ et $\chi_{24}$ avec la SHG, soit :

$$
\begin{aligned}
& \left|\chi_{31}\left(\omega_{1}^{+}=\omega_{3}^{-}-\omega_{2}^{-}\right)\right|=\left|\frac{\chi_{e f f}\left(\omega_{1}^{+}=\omega_{3}^{-}-\omega_{2}^{-}, \phi=90^{\circ}\right)}{F_{31}\left(\omega_{1}^{+}=\omega_{3}^{-}-\omega_{2}^{-}, \phi=90^{\circ}\right)}\right| \\
& \left|\chi_{32}\left(\omega_{1}^{+}=\omega_{3}^{-}-\omega_{2}^{-}\right)\right|=\left|\frac{\chi_{e f f}\left(\omega_{1}^{+}=\omega_{3}^{-}-\omega_{2}^{-}, \phi=0^{\circ}\right)}{F_{32}\left(\omega_{1}^{+}=\omega_{3}^{-}-\omega_{2}^{-}, \phi=0^{\circ}\right)}\right|
\end{aligned}
$$

où les exposants + et - sont relatifs aux modes propres. 


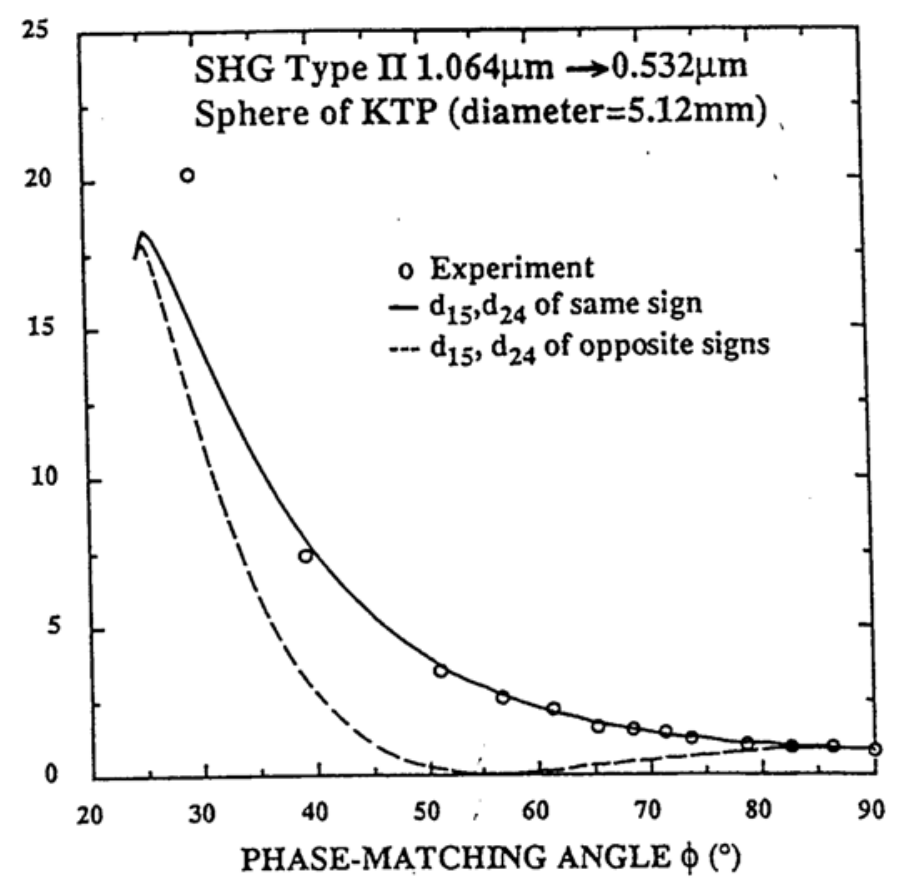

FIG. 20. Puissance harmonique à $0,532 \mu \mathrm{m}$ en fonction de la coordonnée sphérique $\phi$ des directions d'accord de phase de la SHG de type II. Les courbes correspondent au calcul dans les deux hypothèses de signes relatifs [18].

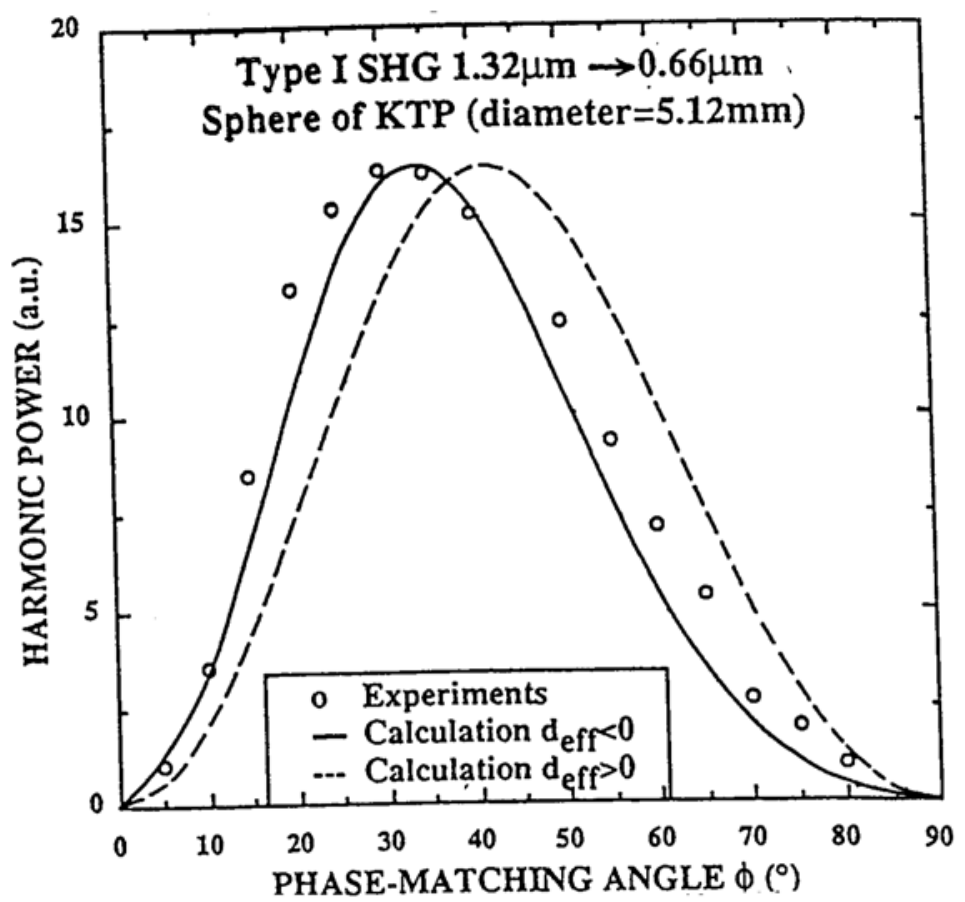

FIG 21. Puissance harmonique à $0,66 \mu \mathrm{m}$ en fonction de la coordonnée sphérique $\phi$ des directions d'accord de phase de la SHG de type I. Les courbes correspondent au calcul dans les deux hypothèses de signes [18]. 
L'idéal est de disposer d'une source laser accordable et de pouvoir trouver une SHG et une DFG qui soient en accord de phase tel que $\omega_{1}$ soit identique à $2 \omega$. Il n'est évidemment pas nécessaire que ces deux interactions soient en accord de phase dans la même direction. Ainsi $\chi_{15}, \chi_{24}, \chi_{31}$ et $\chi_{32}$ seront connus à la même longueur d'onde. Si les mesures ne sont pas réalisées à $\omega_{1}=2 \omega$, il est néanmoins possible de connaître tous les coefficients à la même longueur d'onde en utilisant la relation de Miller qui est une bonne approximation dans la plus part des cas. Elle relie la dispersion de $\chi^{(2)}$ à celle de l'indice de réfraction, soit :

$$
\chi_{i j k}\left(\omega_{c}=\omega_{a} \pm \omega_{b}\right)=\delta_{i j k}\left[1-n_{i i}^{2}\left(\omega_{c}\right)\right]\left[1-n_{j j}^{2}\left(\omega_{a}\right)\right]\left[1-n_{k k}^{2}\left(\omega_{b}\right)\right]
$$

où $n_{i i}, n_{j j}$ et $n_{k k}$ sont les indices principaux de réfraction $n_{x}, n_{y}$ ou $n_{z}$ et $\delta_{i j k}$ l'indice de Miller indépendant de $\omega$.

\subsubsection{Bilan de la méthode en accord de phase}

L'intérêt de l'accord de phase est qu'il permet de déterminer la valeur absolue, avec une très bonne précision, et le signe relatif des coefficients du tenseur $\chi^{(2)}$ qui peuvent être mesurés de façon indépendante. Pour ces coefficients, la précision de mesure est meilleure que dans le cas du hors accord de phase car les rendements de conversion sont supérieurs : d'une part il n'y a généralement pas de problème de détection et d'autre part il est possible de travailler en régime continu ce qui facilite le calcul du rendement de conversion. La détermination du signe relatif est également beaucoup plus facile si la méthode de la sphère est utilisée. Pour la valeur absolue, il est par contre préférable de faire les mesures de rendement de conversion avec des lames minces dont l'épaisseur est inférieure à la longueur de Rayleigh, de façon à travailler en onde plane. Une précision de $5 \%$ est accessible avec des lames minces, alors qu'elle est limitée à $10 \%$ avec une sphère du fait de l'incertitude supplémentaire sur le profil longitudinal du faisceau fondamental dans le cristal [19].

L'inconvénient de l'accord de phase est qu'il impose les configurations de polarisation, de sorte que certains coefficients non linéaires sont mesurés de façon indirecte en se servant des coefficients mesurés de façon indépendante et moyennant l'approximation de Kleinmann. Il est évident que leur détermination sera d'autant plus difficile et d'autant moins précise qu'ils seront nombreux à contribuer au coefficient effectif.

\section{AUTRES CARACTÉRISATIONS IMPORTANTES DES CRISTAUX}

Les propriétés optiques dont l'exploitation est recherchée, ici l'accord de phase et la non linéarité quadratique, ne sont pas les seules qu'il est nécessaire de caractériser en vue de l'utilisation d'un nouveau cristal.

Les coefficients d'absorption linéaires et non linéaires, qui sont reliés aux parties imaginaires de la susceptibilité électrique du premier ordre et du troisième ordre respectivement, sont des données importantes qui viennent souvent restreindre le domaine spectral d'utilisation du cristal. Par exemple, une absorption linéaire supérieure à $5 \% \mathrm{~cm}^{-1}$ viendra parasiter notablement la conversion de fréquence en créant un échauffement. Cette élévation de température entraînera un désaccord de phase d'autant plus important que la tolérance thermique sera faible. La tolérance thermique étant directement liée à la variation thermique des indices de réfraction, il est donc important de mesurer les coefficients thermo-optiques, $\partial n / \partial T$, en fonction de la longueur d'onde. Le lecteur est invité à se reporter au chapitre rédigé par Jacques Mangin dans ce même ouvrage. L'élévation locale de la température peut également causer des déformations mécaniques de volume et de surface pouvant se traduire par des fractures. Outre une caractérisation directe de ce phénomène, il peut être utile de connaître les coefficients élastiques, de dilatation thermique et de conductivité thermique. L'absorption non linéaire à deux photons peut également jouer un rôle important dans la formation de centres colorés qui vont contribuer à élever considérablement l'absorption linéaire. 
D'autre part, une cartographie transverse du rendement de conversion ou de la tolérance angulaire va donner une information sur l'homogénéité spatiale de la qualité du cristal. La figure 22 montre une cartographie transverse du rendement de conversion de SHG dans un cristal de ppKTP : l'homogénéité est bonne avec une déviation standard de seulement $10 \%$ par rapport à la valeur moyenne [20]. Sur ce type d'échantillon, les variations de rendement sont liées à des défauts d'inversion de domaine; le résultat présenté figure 22 est remarquable pour une hauteur de domaine aussi importante. La cartographie de la figure 23 montre que le cristal de $\mathrm{KTiOP}_{0,2} \mathrm{As}_{0,8} \mathrm{O}_{4}$ considéré ici présente une forte hétérogénéité compositionnelle : les propriétés d'accord de phase changent d'un point à l'autre du fait d'une variation du rapport de concentration $[\mathrm{P}] /[\mathrm{As}]$. La caractérisation de l'onde signal émise par génération paramétrique peut également renseigner sur l'homogénéité du cristal. C'est ce qui a été fait avec un cristal de ppLN pour lequel la longueur d'onde d'émission $\lambda_{\mathrm{S}}$, la largeur spectrale $\Delta \lambda_{\mathrm{S}}$ et la puissance associée $P_{\mathrm{S}}$ ont été mesurées en fonction de la position latérale du faisceau pompe. Les résultats sont donnés figure 24 . Les très faibles fluctuations attestent de la très bonne régularité de l'épaisseur et de la succession des domaines, ce qui est remarquable pour un cristal aussi large.

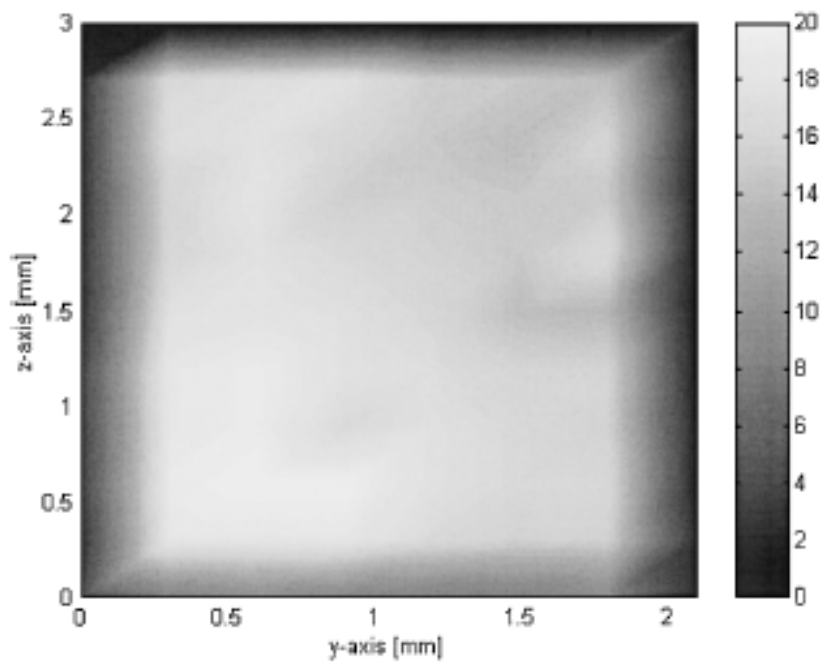

FIG. 22. Cartographie transverse du rendement de SHG 1,064 $\mu \mathrm{m} \rightarrow 0,532 \mu \mathrm{m}$ dans un cristal de ppKTP [20].

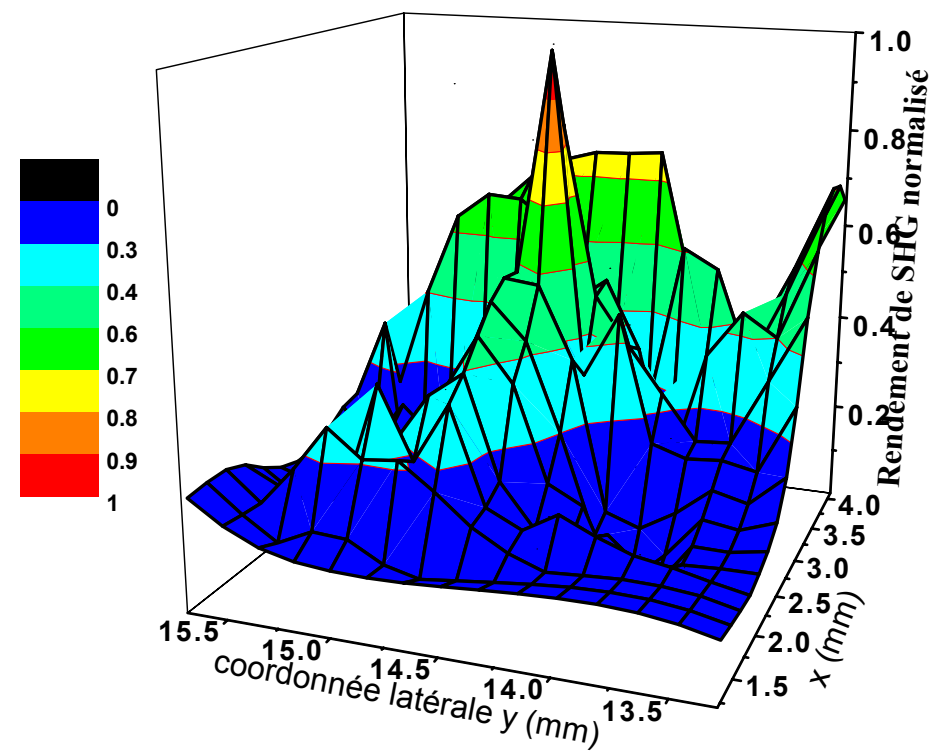

FIG. 23. Cartographie transverse du rendement de SHG de type II $1,064 \mu \mathrm{m} \rightarrow 0,532 \mu \mathrm{m}$ dans un cristal de solution solide $\mathrm{KTiOP}_{0,2} \mathrm{As}_{0,8} \mathrm{O}_{4}$ non homogène. 


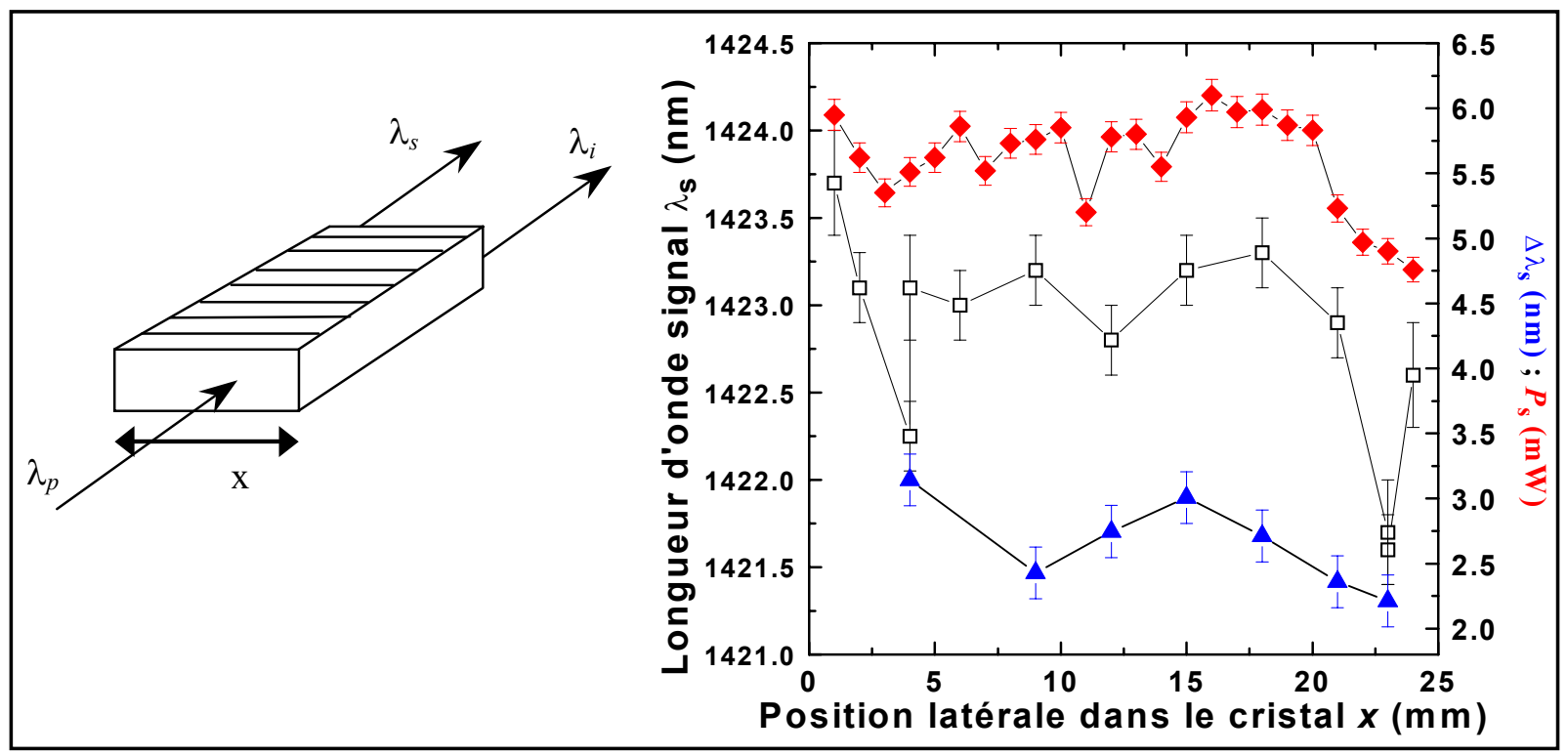

FIG. 24. Caractérisation de l'homogénéité d'un cristal de ppLN en situation de génération paramétrique $\left(\frac{1}{\lambda_{i}}+\frac{1}{\lambda_{s}}=\frac{1}{1,064 \mu \mathrm{m}}\right) . \lambda_{\mathrm{s}}$ longueur d'onde du signal généré, $\Delta \lambda_{\mathrm{s}}$ largeur spectrale du signal, $\mathrm{P}_{\mathrm{s}}$ puissance du signal généré pour une puissance incidente de $42 \mathrm{~mW}$ à $1,064 \mu \mathrm{m}$.

\section{CARACTÉRISTIQUES DES PRINCIPAUX CRISTAUX CONVERTISSEURS DE FRÉQUENCE}

\begin{tabular}{|c|c|c|c|c|c|}
\hline Cristal & $\begin{array}{c}\mathrm{KH}_{2} \mathrm{PO}_{4} \\
\text { (KDP) }\end{array}$ & $\begin{array}{c}\beta-\mathrm{BaB}_{2} \mathrm{O}_{4} \\
(\mathrm{BBO})\end{array}$ & $\begin{array}{l}\mathrm{KTiOPO}_{4} \\
\text { (KTP) }\end{array}$ & $\begin{array}{l}\text { Periodically } \\
\text { poled LiNbO } \\
(\text { ppLN })\end{array}$ & $\mathrm{AgGaSe}_{2}$ \\
\hline Groupe de symétrie & $\overline{4} 2 m$ & $3 \mathrm{~m}$ & $\mathbf{m m 2}$ & $3 \mathbf{m}$ & $\overline{4} 2 m$ \\
\hline Transparence $(\mu \mathrm{m})$ & $0,176-1,7$ & $0,198-2,6$ & $0,35-4,5$ & $0,33-5,5$ & $0,8-18$ \\
\hline Longueur possible (mm) & 500 & 30 & 30 & 50 (épaisseur < 1 mm) & 40 \\
\hline $\begin{array}{c}\text { Seuil de dommage } \\
\text { surfacique }\left(\mathrm{GW} / \mathrm{cm}^{2}\right)\end{array}$ & $\begin{array}{l}>10(1 \mathrm{~ns} \text { à } 1,06 \mu \mathrm{m}) \\
17(0,2 \mathrm{~ns} \text { à } 0,53 \mu \mathrm{m})\end{array}$ & $\begin{array}{c}13,5(1 \mathrm{~ns} \text { à } 1,06 \mu \mathrm{m}) \\
19(8 \mathrm{~ns} \text { à } 0,355 \mu \mathrm{m})\end{array}$ & $\begin{array}{l}9-20(1 \mathrm{~ns} \text { à } 1,06 \mu \mathrm{m}) \\
>2(10 \mathrm{~ns} \text { à } 0,5 \mu \mathrm{m})\end{array}$ & $\begin{array}{c}15-20(30 \mathrm{~ns} \text { à } \\
1,06 \mu \mathrm{m})\end{array}$ & $\begin{array}{l}0,02(10 \mathrm{~ns} ; 10,6 \mu \mathrm{m}) \\
0,01-0,04(50 \mathrm{~ns} ; 2 \mu \mathrm{m})\end{array}$ \\
\hline Remarques & hygroscopique & $\begin{array}{l}\text { sensible à l'eau } \\
\text { (polissage difficile) }\end{array}$ & $\begin{array}{c}\text { dommage } \\
\text { photochromique } \\
\text { à } 0,53 \mu \mathrm{m}\end{array}$ & \begin{tabular}{|c|}
$\begin{array}{c}\text { dommage } \\
\text { photoréfractif dans } \\
\text { le visible }\end{array}$ \\
\end{tabular} & $\begin{array}{c}\text { sensible à l'eau } \\
\text { lentille } \\
\text { thermique }\end{array}$ \\
\hline Interaction & $\begin{array}{c}1,05+0,52 \rightarrow 0,35 \mu \mathrm{m} \\
\text { (THG indirecte) }\end{array}$ & $\begin{array}{c}0,355 \mu \mathrm{m} \rightarrow \lambda_{\mathrm{s}}+\lambda_{\mathrm{i}} \\
(\text { OPO) } \\
410 \mathrm{~nm}<\lambda_{\mathrm{s}}<700 \mathrm{~nm}\end{array}$ & $\begin{array}{c}1,064 \rightarrow 0,532 \mu \mathrm{m} \\
(\mathrm{SHG})\end{array}$ & $\begin{array}{c}1,064 \mu \mathrm{m} \rightarrow \lambda_{\mathrm{s}}+\lambda_{\mathrm{i}} \\
(\mathrm{OPO}) \\
1,36 \mu \mathrm{m}<\lambda_{\mathrm{s}}<1,98 \mu \mathrm{m}\end{array}$ & $\begin{array}{c}10,2 \rightarrow 5,1 \mu \mathrm{m} \\
\text { (SHG) }\end{array}$ \\
\hline $\begin{array}{c}\text { Type d'accord de } \\
\text { phase }\end{array}$ & II & I & II & $\begin{array}{c}\text { QAP, } 3 \text { ondes } \\
\text { extraordinaires }\end{array}$ & I \\
\hline Direction $\theta ; \phi\left(^{\circ}\right)$ & $59 ; 0$ & $24<\theta<33 ; 60$ & $90 ; 23$ & $90 ; 0$ & $54 ; 45$ \\
\hline$\chi_{\text {eff }}(\mathrm{pm} / \mathrm{V})$ & 0,75 & $0,65<\chi_{\text {eff }}<1,5$ & 6,4 & 45 & 54 \\
\hline Double réfraction $\rho\left(^{\circ}\right)$ & 1,5 & $3,5<\rho<4,2$ & $\mathbf{0 , 2 7}$ & $\mathbf{0}$ & 0,66 \\
\hline $\begin{array}{l}\text { Acceptance angulaire } \\
L . \Delta \alpha(\text { mrad.cm })\end{array}$ & 1,2 & $034<<0.29$ & 9,0 & & 17 \\
\hline $\begin{array}{l}\text { Efficacités typiques } \\
\text { (caracteristiques du } \\
\text { laser de pompe) }\end{array}$ & $\begin{array}{c}70 \% \text { (du } 1,05 \mu \mathrm{m} \\
\text { dans } 2 \text { cristaux pour } \\
\text { SHG et THG ; } 2,5 \mathrm{~ns} \text {, } \\
120 \mathrm{~kJ} / \mathrm{impulsion})\end{array}$ & $\begin{array}{c}\eta_{\mathrm{s}}=17 \% ; \eta_{\mathrm{i}}=5 \% \\
(10 \mathrm{~ns} ; 10 \mathrm{~Hz} ; \\
180 \mathrm{~mJ} / \mathrm{impulsion})\end{array}$ & $\begin{array}{c}\eta_{S H G}^{a v}=80 \% \\
(\mathbf{8 n s} ; \mathbf{1 0 H z} ; \\
\text { 900mJ/impulsion) }\end{array}$ & $\begin{array}{c}\text { Seuil } 6 \mu \mathrm{J} / \text { impulsion } \\
(7 \mathrm{~ns} ; 1 \mathrm{kHz})\end{array}$ & \\
\hline
\end{tabular}

TAB. III. Comparaison des principaux cristaux convertisseurs de fréquences. 
Les principaux paramètres caractérisant les quatre cristaux les plus utilisés dans les dispositifs commercialisés sont rassemblés dans le tableau III.

\section{CONCLUSION}

La maîtrise et le développement des méthodes de mesure des propriétés optiques non linéaires de conversion de fréquence conditionnent l'efficacité d'une recherche pragmatique en ingénierie des cristaux et en développement de dispositifs. Cette recherche est toujours d'actualité compte tenu des applications toujours plus nombreuses.

\section{BIBLIOGRAPHIE}

[1] N. Bloembergen, Nonlinear optics, W.A. Benjamin, New-York (1965).

[2] P.N. Butcher et D. Cotter, The Elements of Nonlinear Optics, Cambridge University Press, Cambridge (1990).

[3] B. Boulanger et J.P. Fève, in Wiley Encyclopedia of Electrical and Electronics Engineering, J. G. Webster Ed. (1999), Vol. 15, 278.

[4] J.Y. Courtois, in Les lasers et leurs applications scientifiques et médicales, C. Fabre et J.P. Pocholle Eds. (Editions de Physique, Paris, 1996), Vol. 4. Version électronique : http://dx.doi.org/10.1051/bib-sfo:2002039.

[5] B. Boulanger et J. Zyss, in International tables for cristallography, A. Authier Ed. (International Union of Cristallography, Kluwert Academic, Dordrecht, 2002), Vol. D : Physical Properties of Crystals.

[6] N. Bloembergen, Physical Review, 128 (1962) 606.

[7] M.M. Fejer, G.A. Magel, D.H. Jundt et R.L. Byer, IEEE J. Quantum Electron. 28 (1992) 2631.

[8] J. Zyss, Molecular Nonlinear Optics: Materials, Physics and Devices. Academic Press, NewYork (1994).

[9] D.A. Roberts, Appl. Opt. 35 (1996) 4677.

[10] J.P. Fève, B. Boulanger et G. Marnier, Opt. Commun. 99 (1993) 284.

[11] S.K. Kurtz et T.T. Perry, J. Appl. Phys. 39 (1968) 3798.

[12] S.P. Velsko, Soc. Photo-Opt. Instrum. Eng. Conf. Laser Nonlinear Opt. Eng. 28 (1989) 76.

[13] B. Boulanger, J.P. Fève, G. Marnier et B. Ménaert, Pure Appl. Opt. 7 (1998) 239.

[14] Y. Guillien, B. Ménaert, J.P. Fève, P. Segonds, J. Douady, B. Boulanger et O. Pacaud, Opt. Materials (à paraître).

[15] B. Boulanger et G. Marnier, J. Phys.: Condens. Matter 3 (1991) 8327.

[16] I. Shoji, T. Kondo, A. Kitamoto, M. Shirane et R. Ito, J. Opt. Soc. Am. B 14 (1997) 2268.

[17] W.N. Hermann et L.M. Hayden, J. Opt. Soc. Am. B 12 (1995) 416.

[18] B. Boulanger, J.P. Fève, G. Marnier, B. Ménaert, X. Cabirol, P. Villeval et C. Bonnin, J. Opt. Soc. Am. B 11 (1994) 750.

[19] B. Boulanger, J.P. Fève, G. Marnier, C. Bonnin, P. Villeval et J.J. Zondy, J. Opt. Soc. Am. B 14 (1997) 1380.

[20] J. Hellström, V. Pasiskevicius, H. Karlsson et F. Laurel, Opt. Lett. 25 (2000) 174. 